\title{
Digital Wood: Computational Grain Realignment
}

By

\section{Steven Schuhmann}

\section{A thesis submitted to the Faculty of Graduate and Postdoctoral Affairs in partial fulfillment of the requirements for the degree of}

\section{Master of Architecture}

Carleton University

Ottawa, Ontario

(C) 2016

Steven Schuhmann 


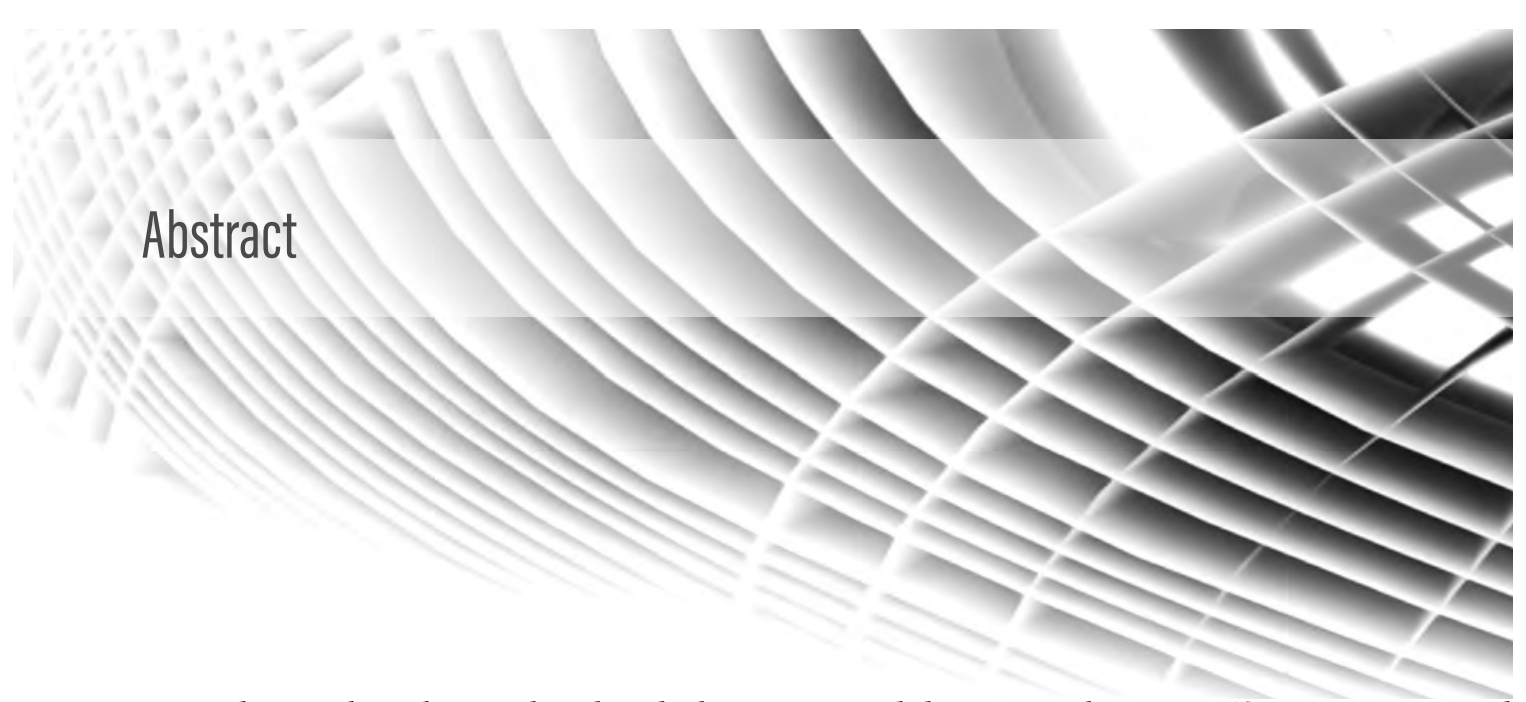

Digital Wood seeks to develop lighter, more delicate, and more efficient structural framing members by computationally realigning wood grain to the corresponding principle stress lines acting within the member while it's under load. This will be achieved by utilizing computational analysis and design software in conjunction with digital milling tools to produce molds, jigs and formwork required for fabrication. Once fabricated, the resulting framing member will retain the performance properties of the original, but in a fraction of the material and thus a fraction of the weight. This materials research thesis will utilize the intrinsic mechanical properties of wood fibre to produce a superior structural framing system. Digital tools of design and fabrication are capable of not only uncovering these properties, but assisting in precision milling required for grain realignment to be viable option in the construction industry. Through this research, I intend to develop a wood-specific structural design methodology that harnesses the strength properties of wood's anisotropic nature. The structural system developed from this working methodology will carry loads and distribute forces in a manner that closely resembles that which a living tree would also carry loads and distribute forces. Ultimately Digital Wood will explore alternative methods of designing and fabricating structural wood components that utilize the properties of wood grain in the most appropriate manner possible and make a meaningful contribution to both the fields of architecture and structural engineering that affirms the versatility and dependability of wood building products. 


\section{Acknowledgements}

I would like to thank the many people that have helped this project, specifically Prof. Manuel Baez for his insight into the nature of fibrous structures, Prof. Steve Fai of the Carleton Immersive Media Studio for providing digital point cloud files of the project site, Brant Lucik for his expertise in CNC milling practices, and Mark Macguigan and Robert Wood for their combined expertise in all things related to making and for providing a suitable workspace to store all of my prototypes and experiments. This project was only possible because of the combined knowledge and support of these amazing people. Lastly, Digital Wood: Computational Grain Realignment would not have been possible without the guidance and support of my advisor Prof. Johan Voordouw of the Azrieli School of Architecture and Urbanism. The direction he provided ensured the work did not stray from the objectives that were defined early in the term and he constantly reinforced a direct, straightforward approach to writing where clarity and simplicity are valued more than esoteric jargon. Thank you. 


\section{Table of Contents}

Part 1 - Branches

Documenting the design and fabrication of a wood composite framing element using the methods of Computational Grain Realignment developed as a part of this thesis. Here, the theoretical framework and existing knowledge influencing this thesis are outlined along with the computational methodology required to generate files necessary in the digital fabrication of this element. Finally, this chapter provides detailed descriptions and diagrams of the fabrication process required to produce this framing element the initial prototype.

Part 2 - Trees

An extension of Part I - Branches, this chapter outlines the design intention and proposed computational methodologies to adapt the knowledge obtained in Part I for the design and construction of an architectural research pavilion located adjacent to the Architecture building on Carleton University campus. The final design of the pavilion is currently under development.

Part 3 - The Forest

Intended to be read in conjunction with the Parts I and II, Part III - The Forest is a philosophical framework intended to contextualize the thesis research. The Forest examines Heidegger's principle of technological revealing in relation the theories of The New Structuralism. It attempts comment on the integrity of digital design technologies for architecture when utilized to create responsive, informed, and non-deterministic architectural design. 


\section{List of Illustrations}

Fig. 1 - Diagrammatic representation of wood fibre

Fig. 2 - Primary Mechanisms of Tree Growth

11

A: Apical Dominance: Form derived from definitive leader

B: Geotropism: Corrective growth directly against gravity

C: Phototropism: Growth directs itself towards the sun

11

Fig. 3 - Example of reaction wood 11

Fig. 4 - Initial digital prototype design 12

Fig. 5 - Exploded view of multi-ply layers 12

Fig. 6 - Schematic diagram of a utility script 14

Fig. 7 - Diagrammatic representation of the typical finite elements analysis

Fig. 8 - Sequence of structural simulation and analysis

16

Fig. 9 - Mesh description $\quad 17$

Fig. 10 - Methods of producing wood veneer

20

Fig. 12 - The Locator Jig 21

Fig. 13 - Fabricated prototype 23

Fig. 14 - Fabricated Prototype - end detail 24

Fig. 15 - Fabricated Prototype - intersection detail 1 25

Fig. 16 - Fabricated Prototype - intersection detail 2

26

Fig. 17 - Beam / Column integration 27

Fig. 19 - Image of site extracted from digital point cloud model

Fig. 20 - Campus context plan with project location

30

Fig. 21 - Instances of direct solar exposure to the project site 31

Fig. 22 - Average of all solar vectors exposed to the project site

Fig. 24 - Average of solar vectors:

33

Fig. 25 - Implemented as physical forces

Fig. 26 - Schematic diagram of utility script 34

Fig. 27 - Initial shell stress simulation results 34

Fig. 28 - Normal principle stress curves (tensile) $\quad 35$

Fig. 29 - Normal principle stress curves (compression)

Fig. 31 - Compression Level 039

Fig. 32 - Tension Level 0 39

Fig. 33 - Tension Level -1 
Fig. 34 - Compression Level -139

Fig. 35 - Tension Level $+1 \quad 39$

Fig. 36 - Final pavilion form to be fabricated (Northwest perspective) 41

Fig. 37 - Final pavilion form to be fabricated (southwest perspective) 41

Fig. 38 - Final pavilion form to be fabricated (Southeast perspective) 42

Fig. 39 - Final pavilion form to be fabricated (Northeast perspective) 42

Fig. 40 - Plan 43

Fig. 41 - Elevation 44

Fig. 42 - Cross-section 


\section{Chapter I- Branches}

Kandinsky dreamed of "a great city built according to the rules of architecture and then suddenly shaken by a force that defies all calculation". This dream sums up the double aspiration of architecture and architectural theory today and the double challenge of computational design. On the one hand we have the ability to

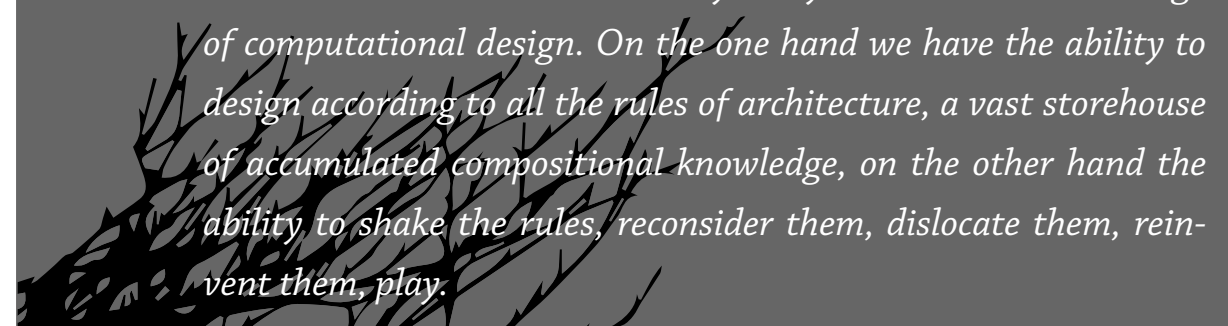

,

- I

Digital Wood. Computational Grain Realignment is research that intends to contribute to thlebody of knowledge pertaining to engineered wood assemblies. It is also participating in the greater discussion of computation and how it will inform the

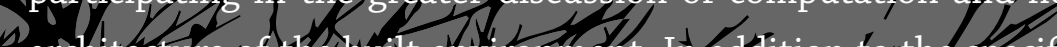
architecture of thes built environment. In addition foythe specific material and fabrication research conducted as part of this thesis the philosophical core is a sequence of - questioning thatencompasses natural systems, the buint enyironment and the com\& puter. Why do these subjects warrant questioning? Never beforehas a technology/like computation revealed so much about the hatural environment and simultaneously

possessed the potential to inform the architecture of the buil environment.

The computer as a design tool has enabled the materialinvestigations and fabrication experiments conducted in this project. Its influence comes from the ability to create models and simulations of natural phenomena that allow architects to respond to contextual forces in an informed capacity beyond that which is possible when designing with analogue methods(Oxmany2012). The Finite Element Method of structural analysis (FEA) is only possiblebecauselof/computation. FEA has served as a fundamental component of this research, notronly/as an analytical tool, but in utilizing the anisotropic properties of wood. Without the analytical and generative capabilities of computation the structural prototypes of this chapter would not be possible. As a modern technology, the computer is allowing us to see aspects of the natural world that we are otherwise blind to.

The revealing that is possible through technology was discussed by Martin Heidegger in The Question Concerning Technology. There are however certain fundamental differences between the technology he discusses and computational technology which must be addressed in order to support the idea of computation as a revealing force. 
Introduction 


\section{Principles, Precedents, Purpose}

Structural timber building components have significantly advanced since the time of primitive log construction. Wood, a seemingly simple material which has historically been treated as homogeneous is in fact composed of cellulose fibres forming a complex internal structure referred to as grain (Fig. 1). The mechanical properties of wood are dependent on the axial direction of the fibres (Fig. 2) however these properties have not been strategically utilized in building construction despite research acknowledging the structural potential when fibre orientation is considered (Mattheck, 1998). This thesis uses computation to realign wood grain such that fibre orientation is optimized in response to internal compressive and tensile stresses acting within a structure. Through careful consideration of wood fibre orientation, this thesis intends to increase strength and structural efficiency while reducing the volume of material required to meet structural requirements. While the objectives of this thesis are quantitative in nature, it is important to note the emerging tectonic logic for timber frame construction that has developed over the course of this thesis exploration. Nondeterministic in nature, the use of computation and digital fabrication techniques ensure not only that structural timber building components continue to advance, but the strengthen the relationship that architecture and structure share.

During the The Siew Fang Chan lecture series hosted at the McGill School of Architecture, Achim Menges, Director of the Institute for Computational Design at the University of Stuttgart in Germany, referred to wood as the "age-old material of the future" (Menges, 2012). Elaborated in Emergent Technologies and Design: Towards a Biological Paradigm for Architecture, Menges was referring to the unique material potential of wood

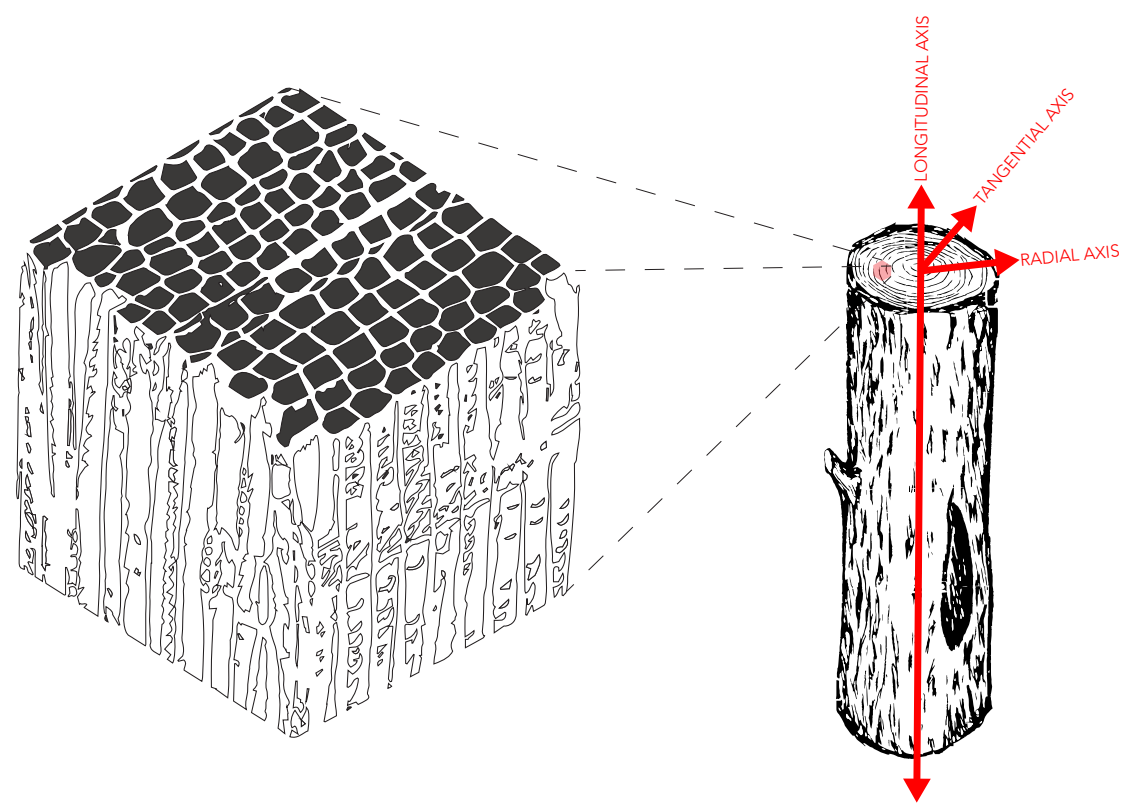

Fig. 1 - Diagrammatic representation of wood fibre (grain). The vertical orientation of the grain (as depicted) corresponds to the longitudinal axis of a piece of timber (depicted right). 
due to its fibrous internal architecture (Menges et al, 2010). The structural capacity of wood is completely due to its anisotropic nature - the internal fibre structure and axial organization. "There is still an enormous unexplored potential in instrumentalizing this towards a positive take on anisotropy and the associated variable behaviour that ensues from it" (Menges et al, 2010), which architects and engineers have yet to fully explore.

Fundamental research by Professor Claus Mattheck, Head of the Department of Biomechanics at the Institute for Materials Research II of the Karlsruhe Research Centre (Karlsruhe, Germany), outlines the design intelligence and mechanical potential of the tree. The extents of Mattheck's research reveal two principles that are applicable to the field of architecture: The Axiom of Uniform Stress, and Force Flow. In his book Design in Nature, Mattheck describes the axiom of uniform stress as a phenomenon whereby the tree grows in a manner that evenly distributes the external forces acting on it in order eliminate localised stresses. The tree achieves a state of equilibrium as it deposits material where it is needed. The second principle of importance to this thesis is that of force flow, which is related to the axiom of uniform stress, but pertains to the internal orientation of the wood fibres relative to the direction of the internal stresses. The orientation of the wood fibre is parallel to the internal principle stresses - a feat that has served as a driving principle in the development of composite prototypes for this thesis. This property is noteworthy because parallel grain wood performs poorly in shear stress and by definition the principle stress trajectories to which the tree grain is deposited are absent of shear stress - the tree is actively adapting to mitigate the presence of shear stress (Mattheck, 1991). Mattheck describes how an 'ecodesign' is not simply the optimization of the external shape, but also of the internal fibre distribution. This relationship of external form and internal structure is profound, but not accessible through traditional design. To analyse these internal properties and optimize their potential computation is required.

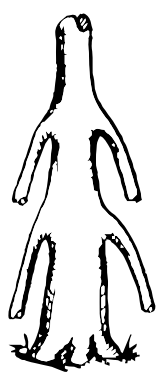

A

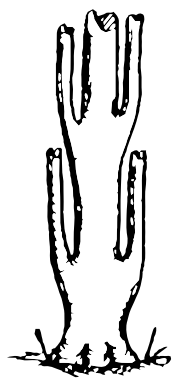

B

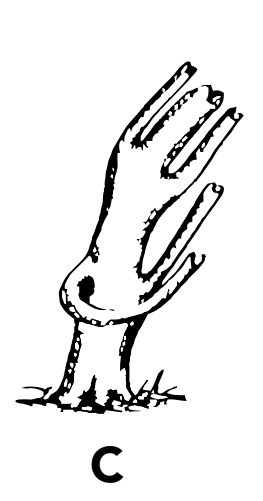

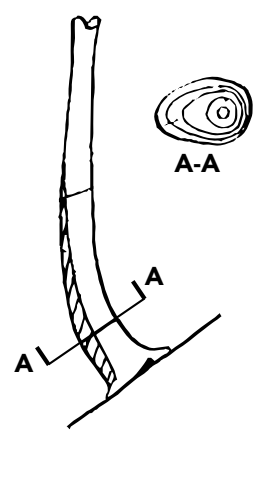

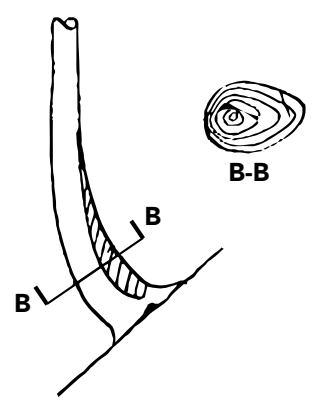

Fig. 3 - Example of reaction wood formation driven by geotropism. Compression wood is formed at the underside of the member $(A-A)$, while tension wood is formed at the top side (B-B).

A: Apical Dominance: Form derived from definitive leader B: Geotropism: Corrective growth directly against gravity

C: Phototropism: Growth directs itself towards the sun 
Digital software such as Rhinoceros, Grasshopper3D and various purpose-built analytical plugins enable the architect to unlock and appropriate the material potential of wood fibre. As Kostas Terzidis states in Expressive form: A Conceptual Approach to Computational Design, architects are now able to explore a full range of physical forces acting on a design through the advances made in computation and ability to model and simulate these forces. (Terzidis, 2003). Computation becomes integral not only for analysis and simulation of real world physics, but also for the design and fabrication of the complex forms and geometries that result from these dynamic simulations - forms and geometries that cannot be constructed via conventional means but rely entirely on the use of digital fabrication technology. The key to unlocking the material potential in wood comes not only from a scientific understanding of the internal fibre structure of the wood, but from developing means to actually fabricate and implement these geometries.

The objective of this thesis exists at the intersection of a complex natural process and contemporary tools of digital analysis, design and fabrication. Existing research and stress testing has demonstrated distinct mechanical properties of wood that are grain-dependent with superior tensile and compressive properties parallel to grain than other orientations. How then can we use this knowledge to inform architectural design and the corresponding structural components? What I hypothesize is Grain Realignment whereby the longitudinal axis of the wood grain is oriented such that it is parallel to the internal tensile and compressive stresses acting within a structure. Force-dependent tree growth and wood fibre orientation, as outlined by both Menges and Mattheck, are intrinsically valuably to architects seeking to advance timber structural components. This property of material efficiency and structural design intelligence presents a compelling case for further analysis and exploration into means by which this natural process can inform both architectural and structural design. The intent of this research is to develop a means of harnessing these properties and using them to inform an interrelated archi-structure, ensuring the design intelligence developed by the living tree is not lost once transformed into timber products used in construction today by conventional milling practices.

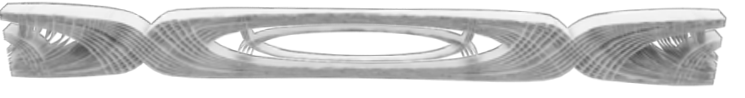

Fig. 4 - Initial digital prototype design. The intention was to create a wood beam that clearly articulates the internal principles stresses acting within the member while a force is applied to it. This scenario utilizes a single point load, centred on the beam.

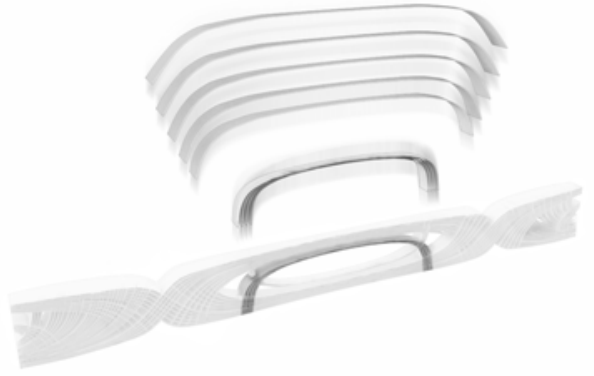

Fig. 5 - Exploded view of multi-ply layers intended to be constructed of Douglas Fir veneer strips. Physical prototyping was attempted, however steam bending was used to assist in achieving the tight curvature required for the veneer laminations. This ultimately rendered the veneer brittle and unfit for CNC milling as steam bending softens the woods lignin and reduces the strength of the wood. The required curvatures were achieved and initially showed promise but the intersecting lap joints could not be milled into the wood. 
Methodology 


\section{Methodology - Analysis}

Computational Grain Realignment begins with the analysis of stress in a structure and locating regions of principle stress - stresses acting in perfect tension or compression. The computational analyses conducted first used simple elements like beams and columns as the test subjects. As the project advanced and the analysis algorithms were further refined the geometry that was analysed became more complex. This project isolated the principle stresses of a structure through finite element analysis (FEA) and extracted curve geometry that was indicative of the internal compressive and tensile forces acting within the structure. Finite element analysis is a computational solver that has the ability to simulate forces acting on an object in predetermined loading and support scenarios. The analysis returns a set of reaction data. The regions of principle normal stress acting within the structure are of specific interest. A series of curves that represent these forces are extracted and subsequently used to inform the fabrication process (detailed in the following chapter). For fabrication purposes the curves representing tensile and compressive forces must be isolated. In this analysis the FEA solver returns raw data that must be sorted, interpreted and appropriated in order to optimize and subsequently design the final structure. The volume of data returned by the FEA solver is directly related to the mesh resolution of the geometry analysed. A mesh is a grid that defines the geometry. Each square of the grid is called a facet. Mesh resolution refers to the number of facets that comprise geometry. Higher resolution mesh geometry will allow the FEA to compute more accurate results but also generates a larger data set to interpret. Computational algorithms become invaluable, as they are able to sort, segregate, split, sift, or extract the data that is of value. The schematic diagram below demonstrates a key utility script developed to sort through and extract the desired FEA data. A utility script is a processing algorithm that is applied to a data set that will retrieve relevant data. Several utility scripts

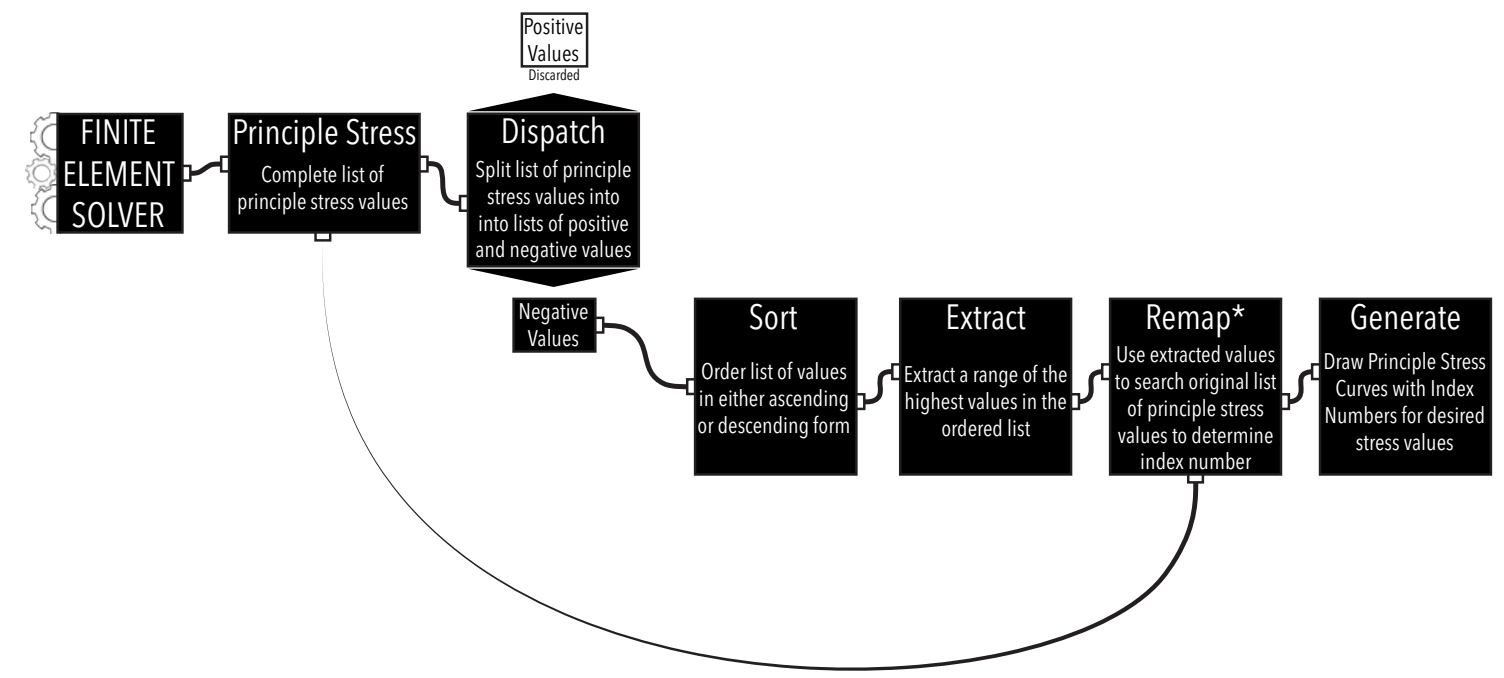

Fig. 6 - Schematic diagram of a utility script used to sort through available principle stress values and return a numerical domain of the highest values. 

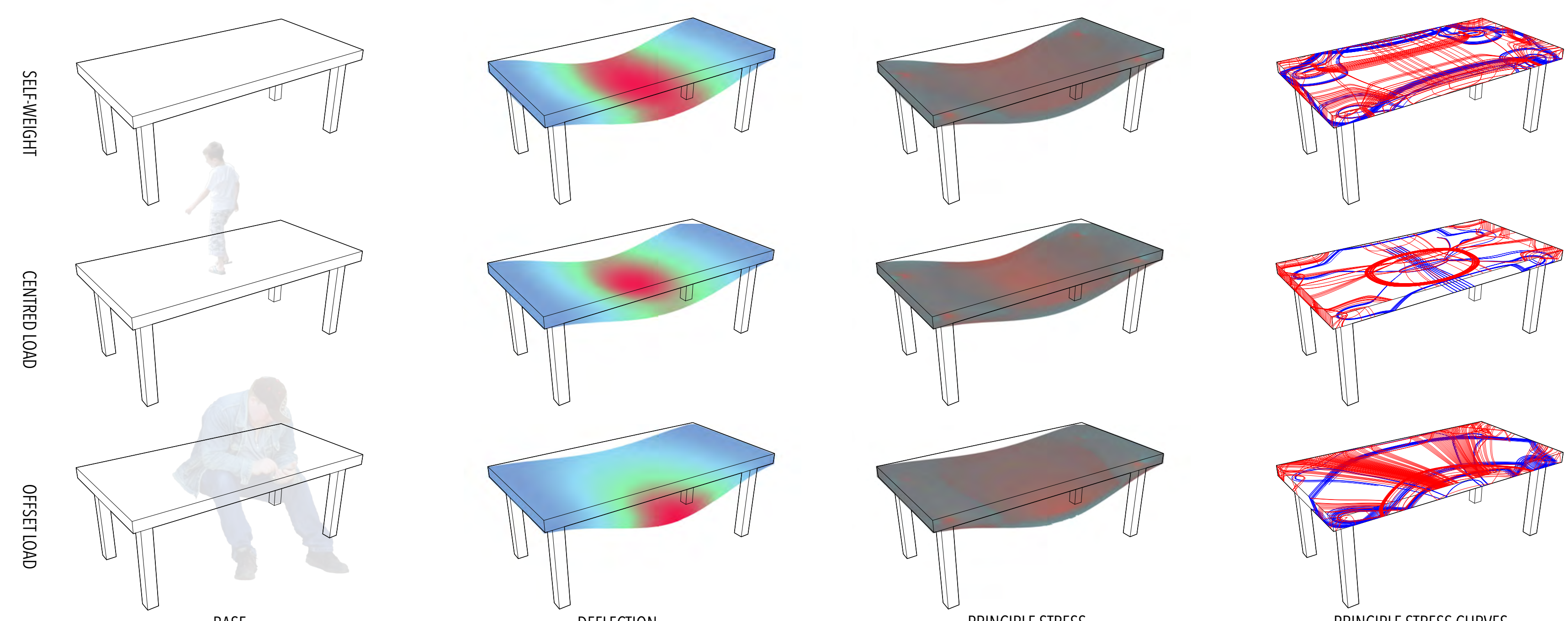

BASE

DEFLECTION

PRINCIPLE STRESS

PRINCIPLE STRESS CURVES 


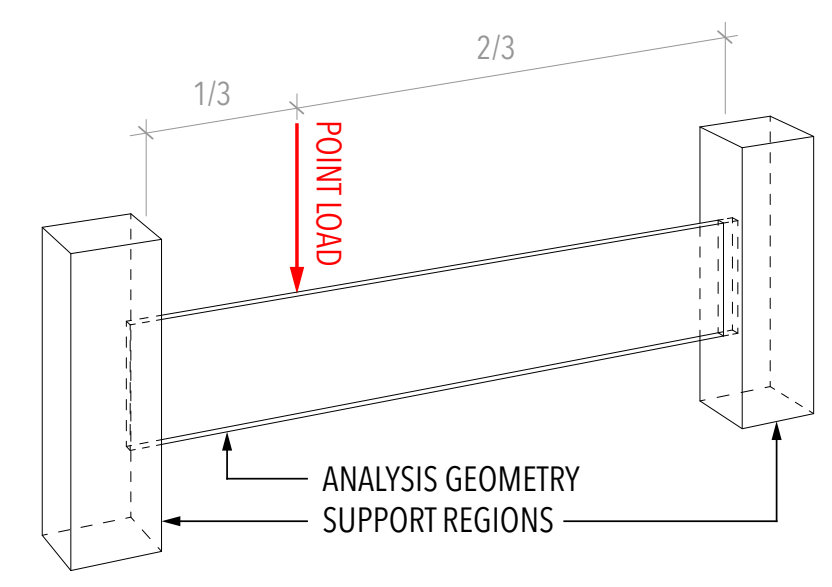

1 - Define Structure and Loading

For the purposes of this initial investigation a simple structural condition is defined. A wood beam spanning $1.2 \mathrm{~m}$ is fixed at each end with supports that resist translation and rotational moments in all directions. I single point load is applied to the beam. It is important to note that the geometry depicted left does not accurately reflect building components in reality, but instead are modelled as required by the FEA solver to compute the reactions.

3

2 - Finite Element Simulation

The FEA solver computes a series of iterations that gradually begin to accurately reflect the structural reactions. The solver has the ability to perpetually compute this structural condition in order to return more accurate data, however a reasonable solution is returned quickly. The digram directly left depicts the anticipated deflection, exaggerated for demonstrative purposes. The diagram at the bottom left of the page outlines the regions of principle stress that are of particular interest for this project. The regions of red indicate compressive stress and the blue/green regions indicate tensile stress

3 - Extracting Principle Stress Geometry

The FEA solver utilized in this investigation is equipped with a function that will generate a set of curve geometry that is representative of the principle stresses generated during the simulation. A very large number of compressive and tensile curves result that must be sorted and interpreted in order to be utilized further.

4 - Organizing Principle Stress Geometry

The goal of this investigation was to isolate the single greatest curves representing principle stress. A sorting algorithm was developed to analyse the list of curve geometry and find the curve that represented the highest principle stresses, shown in the diagram directly right. The processed curve geometry would serve to inform the design and fabrication of the beam prototype that is detailed in the following chapter. 
were developed over the course of this project to not only expedite data processing but also remove human error that will inevitably compound over many repetitive actions.

\section{Methodology - Fabrication}

All prototypes fabricated for this project are wood-composite assemblies. Spruce, pine or fir (SPF) was the desired species for these prototypical fabrications as they are almost exclusively the species of choice for timber building products. Due to availability issues of certain SPF veneer products, alternative species have been substituted. The fabrication strategies developed utilize 3-axis Computer Numerical Control (CNC) milling technology as a means to produce lamination formwork, or jigs, and to machine joinery into fabricated prototypes. Careful consideration was given to sourcing materials and developing fabrication strategies that reflect the capabilities of the structural timber industry, as it exists today in Canada. The aspiration is to develop a successful working methodology that can produce a viable product for the industry to fabricate with only minor adjustments to current practices.

$$
\text { Methodology - Testing }
$$

For the purposes of establishing reliable metrics and to serve as a proof-of-concept, the prototypical assemblies are to be tested at Carleton University's Civil Engineering laboratory (dates pending). Testing will be conducted similar to the stress testing of clear wood typically used to establish the mechanical properties of various wood species. It is my hypothesis that stress testing the prototype produced as a part of this thesis will reveal a superior strengthto-weight ratio when compared to a traditional timber beam.
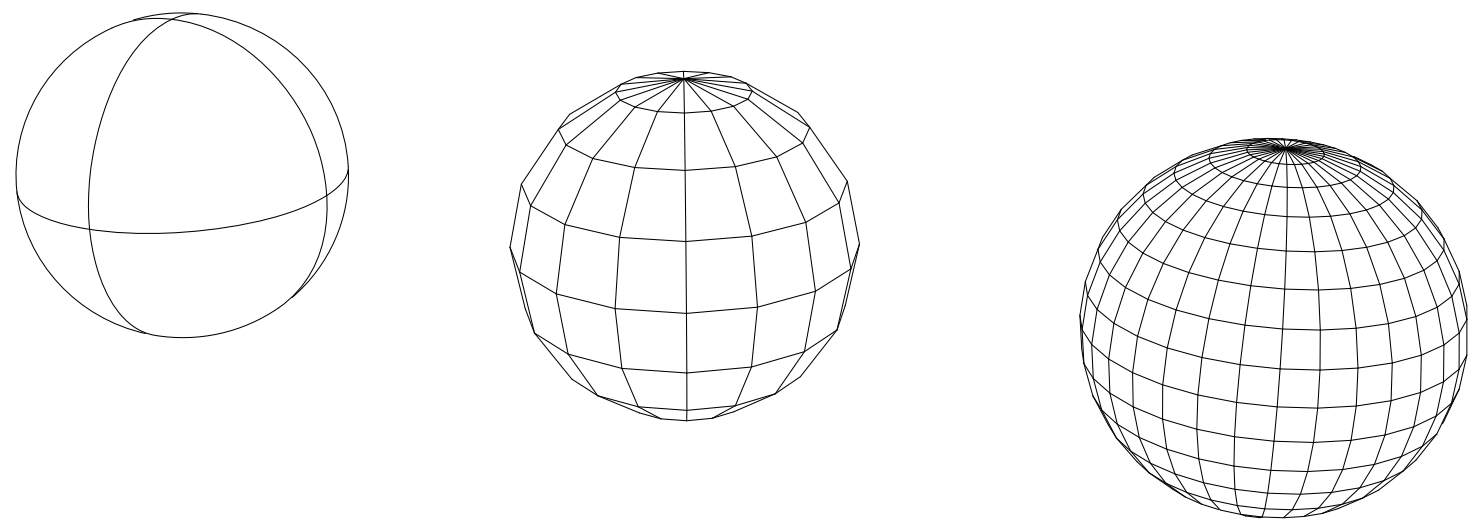

Fig. 9 - Sphere (left), Mesh Sphere 1 (centre), Mesh Sphere 2 (right). Both Mesh Sphere 1 and Mesh Spere 2 describe an identical sphere, however Mesh Sphere 2 consists of more facets than Mesh Sphere 1. 
Fabrication 


\section{Sourcing Materials}

This thesis is informed by the material properties of wood. The search for material types to be used in fabricated prototypes began in the early early stages of this project. Research was conducted into the current state of wood-composite assemblies and laminated (glulam) building products which revealed that wood veneer could serve as the material type of choice to fabricate the structural geometries developed from the analysis conducted in the previous chapter. In reference to engineered wood products that utilize veneer to form solid mass assemblies, Joseph Iano states they were originally developed to overcome the shortcomings of solid wood structural members and had assumed a new importance in an era of conscious sustainability. However, as this thesis intends to address, despite improving upon the shortcomings of solid wood structural members, the wood, as an engineering material, is still structurally under-utilized as no attention is given to the grain orientation relative to the principal stresses acting within the structure.

Initial fabrication experiments utilized hand-milled Douglas Fir veneer strips because this species is most commonly used in laminated wood assemblies for structural elements. Unfortunately Douglas Fir veneer is not commercially available outside of the manufacturing industry making it unattainable at the necessary scale for this thesis. As such, an alternative species was substituted.

Red oak veneer was selected in-place of Douglas Fir. Despite being a hardwood species, Red Oak possesses similar mechanical properties to Douglas Fir and was available in the required quantities. It is the aim of this project to grow to a scale where fabrication experiments may eventually be conducted with construction-grade softwoods. A conscious effort of the fabrication experiments was to utilize materials and processes that are commercially available and well-established in the hopes that with minor adjustments, industry can begin manufacturing alternative engineered wood products using existing materials and infrastructure.
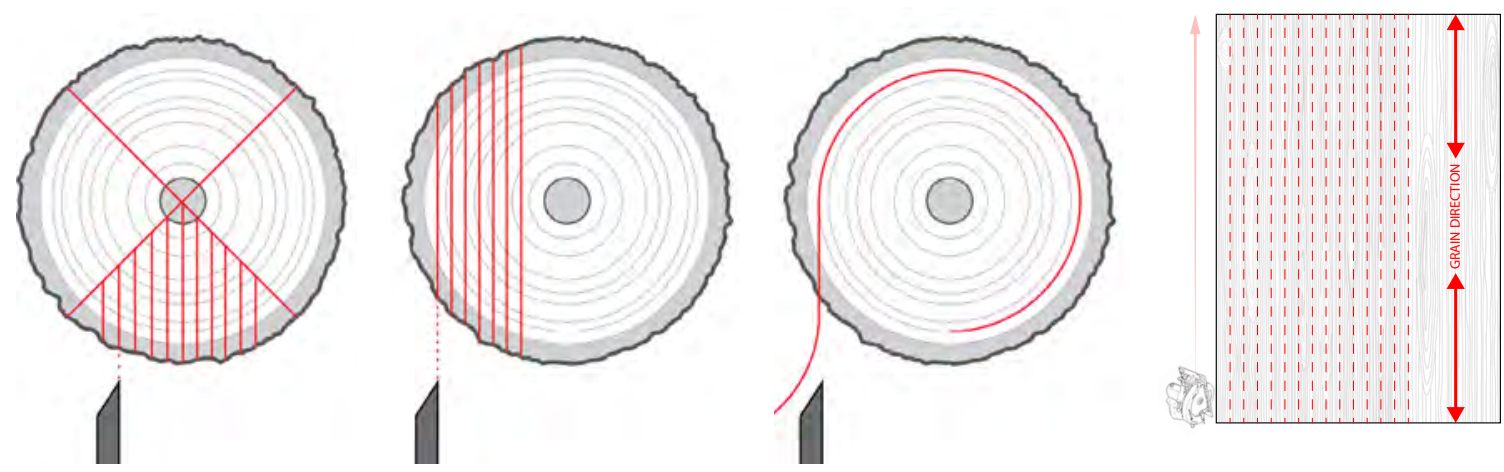

Fig. 10 - Methods of producing wood veneer. From left to right, Quarter Cut, Plain Cut, Rotary Cut. Rotary Cut veneer was selected for the fabrication experiments because the process produces sheets of veneer with uniform grain orientation as outlined in the diagram far right. 
Rotary cut veneer was selected as the material type of choice for the laminated assemblies of this thesis not because of the material efficiency of it's manufacture, but because "the direction of the grain in piece of wood affects every aspect of how we can use it" (Iano et al, 2007). The non-backed veneer is flexible enough to conform to the required curvatures of the geometry created when principle stress is considered in the design of a structural member. This flexibility allows the wood grain to flex and align to the principle normal stresses computed during the finite element simulation and eliminate redundant material present in the structural member not only making it lighter but lending a finesse and elegance reminiscent of biological elements found only in nature.

\section{Lamination Jigs}

The finite element analysis conducted in the previous chapter returned four unique principle stress curves - two in tension and two in compression that measured higher than the thousands of other stress curves present. These four unique, asymmetrical, and curvilinear bands would be fabricated with the assistance of laminations molds (jigs). The geometry required to fabricate the jigs was derived from the same computational process that generated the principle stress bands, demonstrating that finite element analysis can be used not only in the analysis of the new structural members, but also in the fabrication of the new structural members. The geometry for the jigs required a degree of post-processing in order

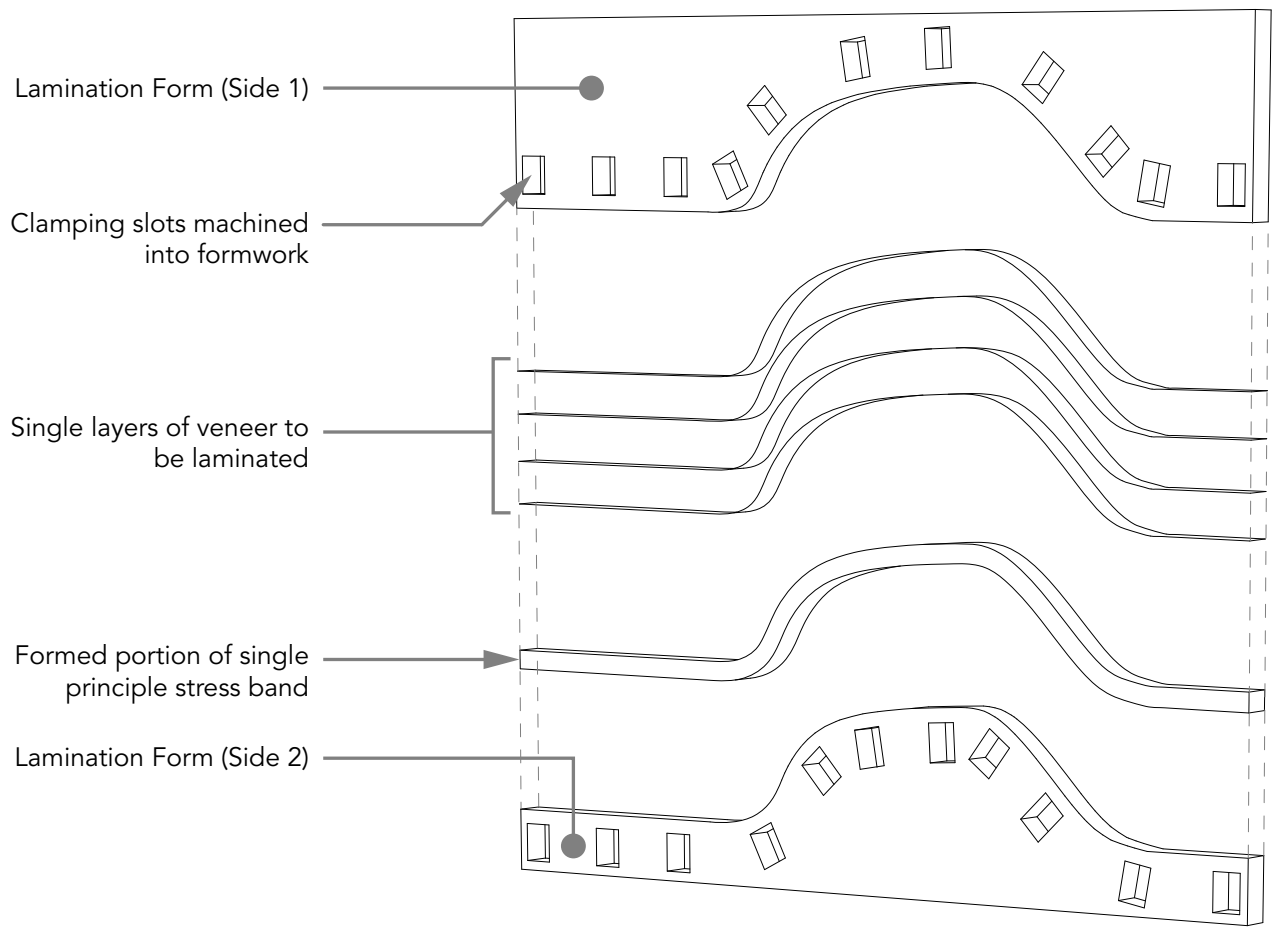

Fig. 11 - One of four lamination jigs required to fabricate the principle stress bands that comprise the beam prototype. 
to prepare the design components for 3-axis Computer Numerical Control (CNC) milling. The post-processing was minimal, consisting primarily of joining open lines to create closed polylines, and in most instances was automated algorithmically through a utility script.

The jigs consisted of two layers of $3 / 4$ " plywood laminated together. Clamping slots were milled at regular intervals along the two clamping faces of the jig to ensure even distribution of clamping pressure during lamination. Each principle stress band consisted of forty individual layers of veneer that were laminated successively in 8-strip intervals. In a manufacturing setting, this process would be automated, however due to the working scale of this lamination process, the work was executed manually in clamping intervals. This would ensure even adhesive distribution and that the adhesive did not lose sufficient moisture before clamping, ensuring the strongest possible bond.

\section{Machining Principle Stress Bands}

Once laminated, the faces of normal principle stress band were machined to create a smooth, even surface in preparation to mill the lap joints. Each band was passed over the jointer followed by several light passes through the surface planer. It was imperative that a single face of each band was passed over the jointer before using the surface planer to ensure a uniform width throughout the length of the band.

\section{The Locator Jig and Milling Lap Joints}

Because the principle stress bands were laminated and subsequently milled with a 3-axis $\mathrm{CNC}$ router, it was imperative to devise a system whereby the items to be milled could be located on the 4'x8' bed of the CNC machine. Conventional subtractive manufacturing

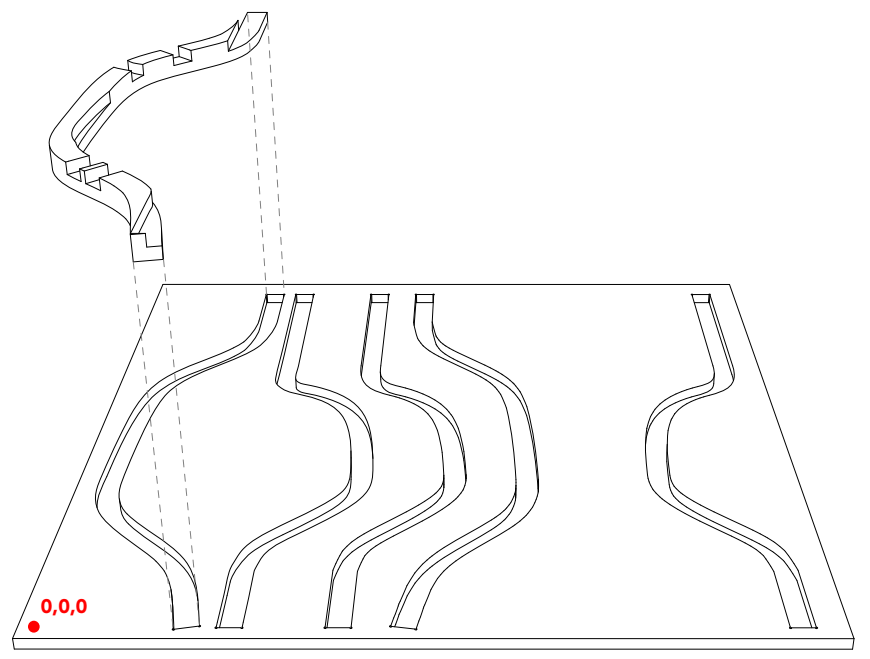

Fig. 12 - The Locator Jig - A single sheet of 3/4" plywood that has profiles of the 4 principle stress bands milled out. This jig serves to secure the work to the CNC bed as well as establish a common origin point with the CNC machine. 
requires that objects are milled from a single piece of larger stock. The reductive nature of this process produces large amounts of waste. This attribute of the fabrication process was undesirable as one of the objectives of this thesis is to develop a system that reduces material waste through digital fabrication. In order to locate the principle stress bands within the limits of the CNC machining bed, a positioning jig, referred to as the "locator jig" was produced.

At the same time the lamination jigs were fabricated, a locator jig was also fabricated with the CNC router. The purpose of the locator jig was to establish a common origin $(0,0,0)$ between the jig and the principle stress bands to be milled. The locator jig also served to secure the bands to the machine table ensuring the lap joints could be safely and accurately milled. While this is not a common practice with subtractive manufacturing, it is within the tolerances and capabilities of the machine if adequate consideration is given to establishing origin points across machining jobs and measures are taken to precisely located objects within the machine bed.

Ultimately, milling the lap joints was well executed, resulting in tight, friction-fit joints and principle stress bands that required clamping pressure in order to assemble given the high tolerance to which they were milled. Additionally, use of the locator jig to mill the lap joints produced an insignificant amount of waste material - only that which was absolutely required to achieve the joint. A simple consideration such as this has the potential to drastically reduce the volume of waste material that would typically result from a reductive manufacturing process. 


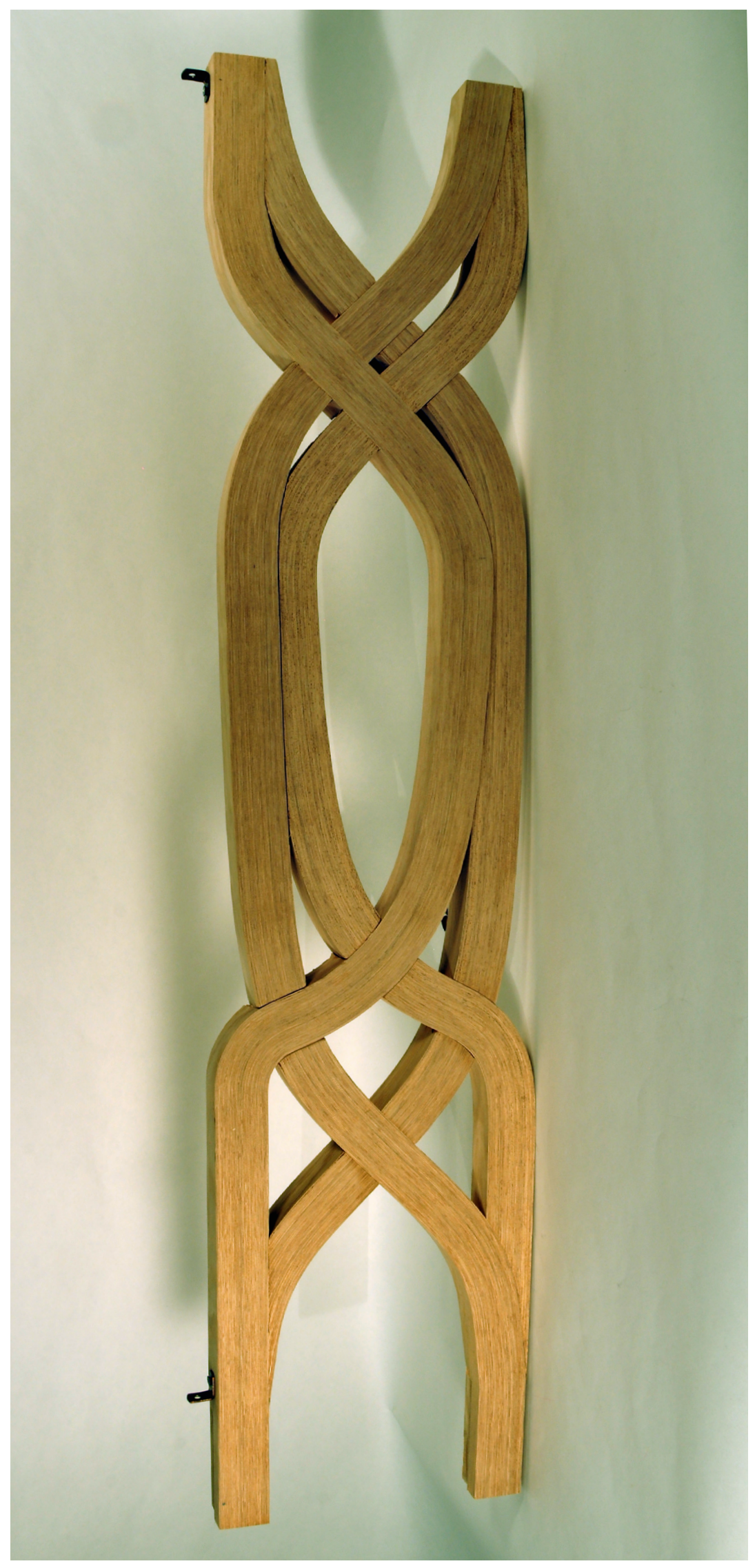

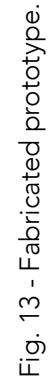




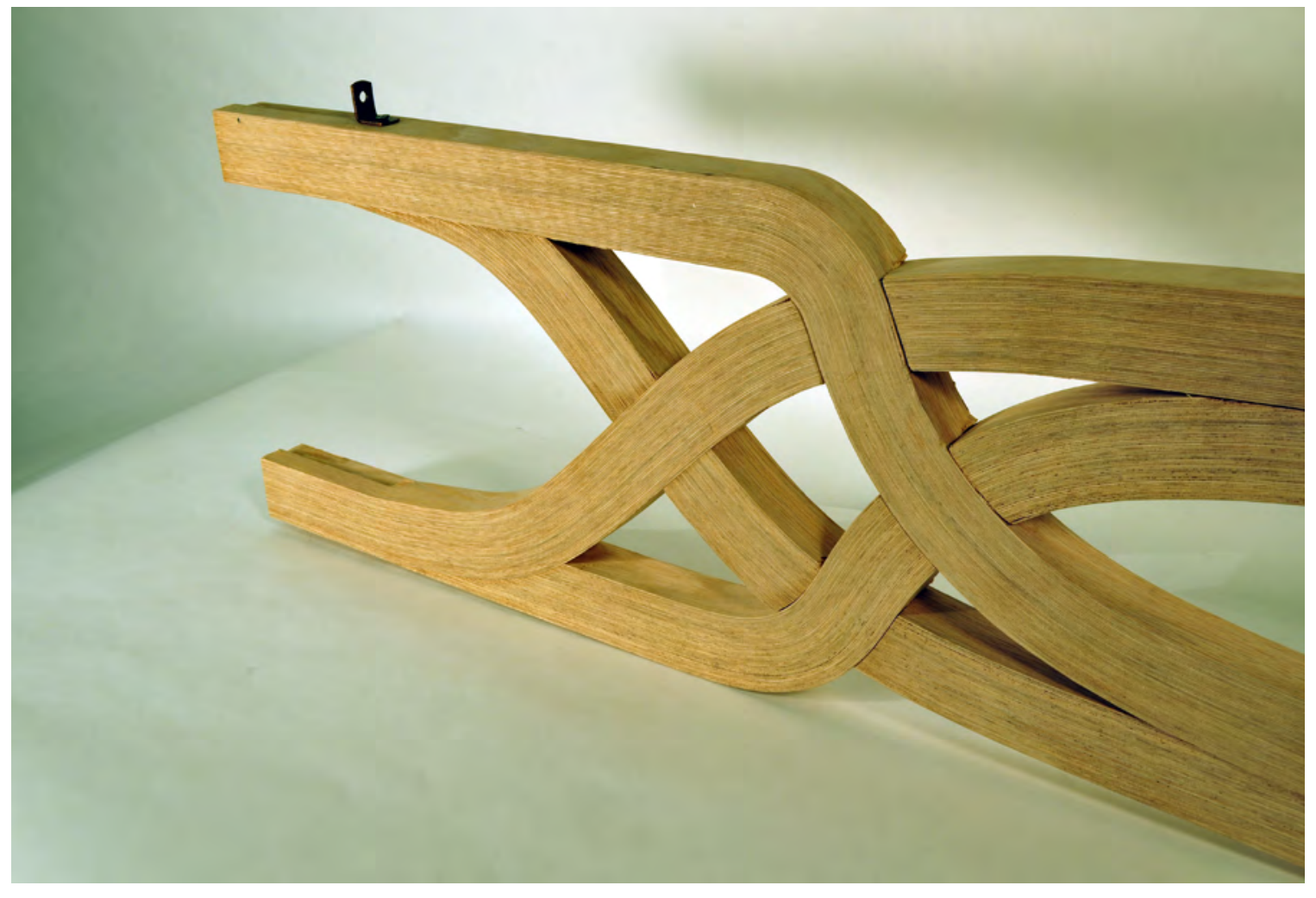

Fig. 14 - Fabricated Prototype - end detail 


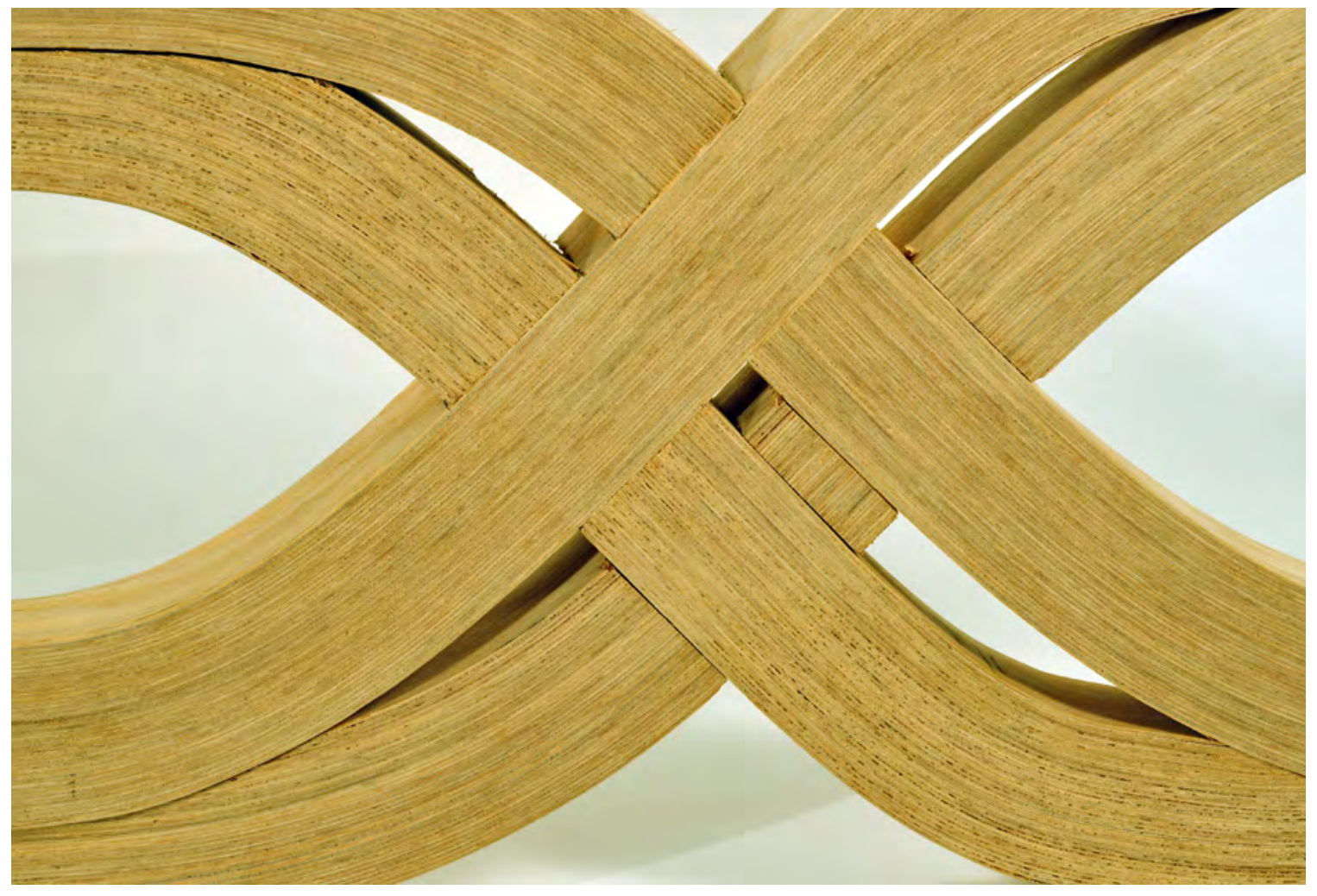

Fig. 15 - Fabricated Prototype - intersection detail 1 


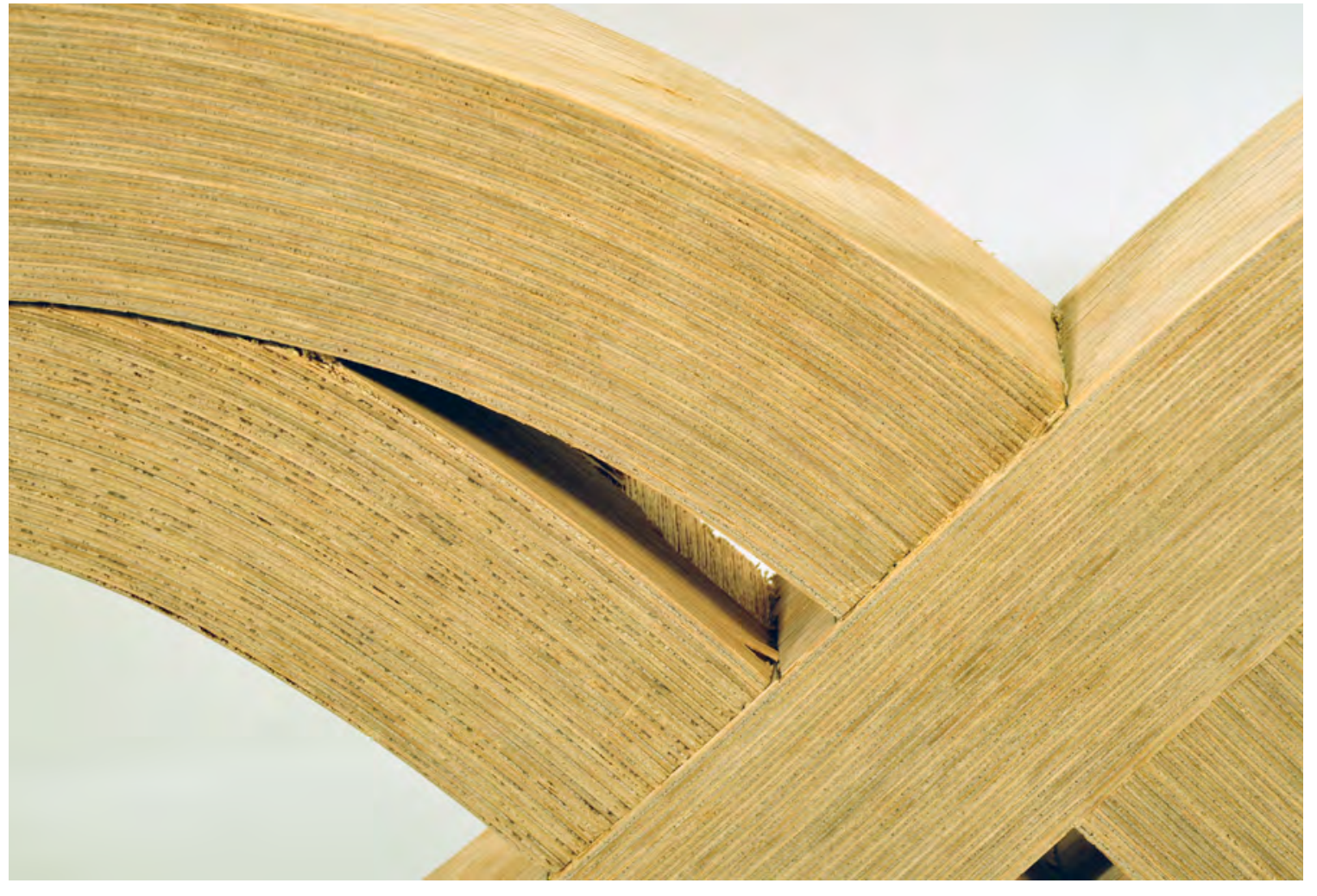

Fig. 16 - Fabricated Prototype - intersection detail 2 


\section{Post-Fabrication Analysis}

While the fabricated beam prototype is considered successful for it's strategic use of material and digital fabrication technology, it is also with its deficiencies that were realized post-fabrication. As research and development continued it became apparent several important considerations for this prototype were overlooked. These include: (1) the thickness of the compressive and tensile stress bands, (2) the order in which the stress bands were assembled and (3) the manner in which a beam such as the prototype of this project would integrate with other structural elements that were designed and fabricated with the same tectonic logic (see below).

The structural behavior of fibre structures, including wood, vary with the tensile and compressive forces that are applied to a structure. Mechanical testing of various wood species has demonstrated that wood, parallel to the grain, is about twice as strong in tension than it is in compression (Tsoumis 169). This would suggest that the tensile and compressive stress bands should have different cross-sectional widths and that the overall material volume of this prototype could be further reduced through a reduction in the width of the tensile stress bands. Determining the ratio of cross-sectional widths of the tensile and compressive stress bands will require further investigation. However, I hypothesize this ratio is related to the comparative metrics for the tensile and compressive strength of wood parallel to the grain.

Wood is stronger in tension than in compression. This is in fact true of all fibre structures in nature as their tuberous cellular composition makes them prone to buckling when subjected to compressive force. Michael Hansel has described the only four known strategies for mitigating compressive stresses in fibre structures found in biological systems:
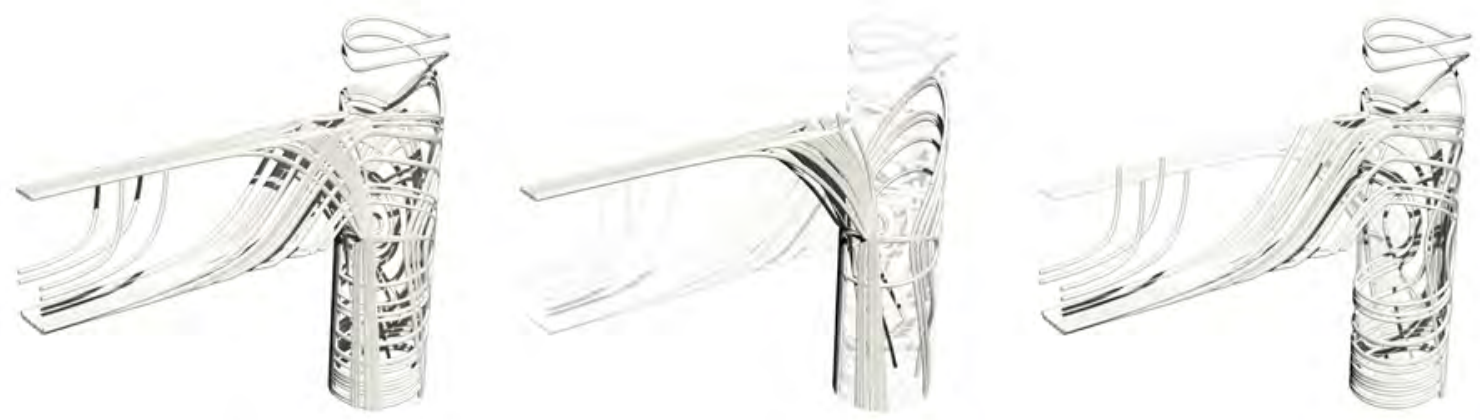

Fig. 17 - Beam / Column integration using Computational Grain Realignment: The same analysis methodology detailed in the previous chapter was applied to the condition of a beam meeting a column. The analysis demonstrated how principle stresses traveling through the beam weave into the column and travel downward. This condition and the manner in which the stress curves are flowing poses new challenges for fabrication. In the event this project were to continue beyond the $\mathrm{M}$. Arch thesis, exploring fabrication strategies related to connections such as these would be a natural progression. 
[I] pre-stress the fibres in tension so that they hardly ever experience compressive loads;

[II] introduce high modulus mineral phases intimately connected to the fibres to help carry compression; [III] heavily cross-link the fibre network to increase lateral stability; [IV] and change the fibre orientation so that compressive loads do not act along the fibres

It was apparent after discovery Hansel's strategies for mitigating compressive forces that with a small modification to the assembly of principle stress bands, a cross-linked network could be established to increase lateral stability and improve the performance of the compressive stress bands. This would require the tensile stress bands to sit at the extents of the structure thus eliminating the potential for the compressive stress band to buckle as it is braced by the tensile stress band at each side (refer to diagram).

The realizations outlined are invaluable as the project advances to the design and fabrication of a small research pavilion. Appropriately sizing the principle stress bands and establishing a cross-linked network in which the tensile framing members are strategically placed to mitigate buckling of the compressive framing members will inform the fabrication methods of the research pavilion.

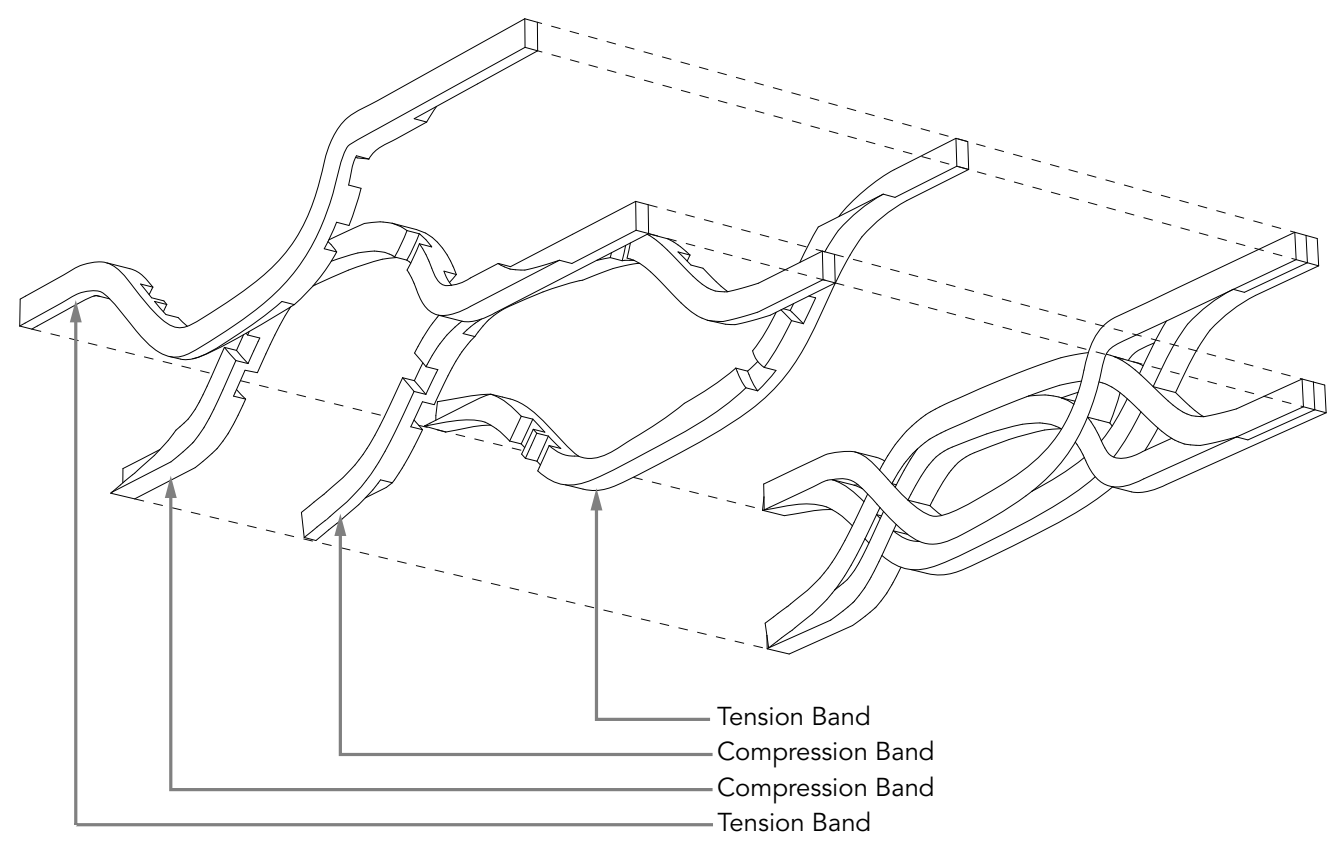

Fig. 18 - Schematic diagram of utility script used to sort through available principle stress values and return a domain of the highest value. This computational logic was developed and implemented to retrieve the highest principle stress values, in 


\section{Chapter II - Trees}

Computation is an invaluable tool for architects because of its ability to model complex natural processes and simulate physical forces. Through computation there is a revealing of the natural world in a true Heideggerian sense but with a single difference; the modern technology that I am discussing is not challenging forth but is revealing aspects of the natural world that we could not understand that we can subsequently harness and utilize to inform the architecture of the built environment. Heidegger differentiates between ancient and modern technology arguing that the latter challenges into existence rather than reveals (Poeisis). His understanding of modern technology did not account for the computer though, and was limited to the advances of combustion technology of his time. Additionally, one may argue that his perception of modern technology was also the result of the mechanistic worldview that served as a fundamental principle of modern philosophy whereby humanity is separated from natuke (Ishida/Furukawa, 2013):

The Heideggerian notion of-revealing should not be negated, but rather expanded to include the Poeisis that is possible thot th computation. Despite Heidegger it is apparent that computation is reveals far more it challenges. This is in fact the entire premise of the New Structuralism and what leading architectural thinkers are describing as a matērials-first approach to design (Toshiko, 2002). Computation also allows for emergence through algorithmic logic and definitions that are modeled on phenomena observed in nature. This is strikingly similar to the notion of Physis Heideger wrote of and has spawned a sub-branch of computational design referred to as Morphogenesis (Menges et al, 2010).

In her examination of digital-désign and digital methods for the production of architecture, Rivka Oxman acknowledges severat compelling aspects of digital media that arefundamentally altering how we conceive-of,-investigate and design for the built environmentiv

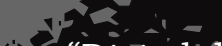

(1) "Digital design thinking is mo

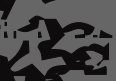

re than simply a set of formal preferences. It is the abandonment of the modernist design ontology that is predicated on formal and typological knowledge (e.g. formal languages, typological classes and generic design,(ete:) It is non-typological, and non deterministic in supporting and preferring the differentiated over the discrete and typological

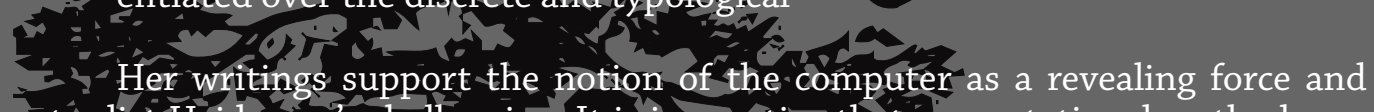
contradict Heidegger's challenging. It is imperative that computational methods are information-based and do not presuppose in the deterministic fashion Heidegger describes of modern technology. Computation employs analytical reasoning and information to formulate design conclusions. Thus, the architecture that emerges of a digital method, of computation, is fundamentally different from the architecture that results of anălógue means.

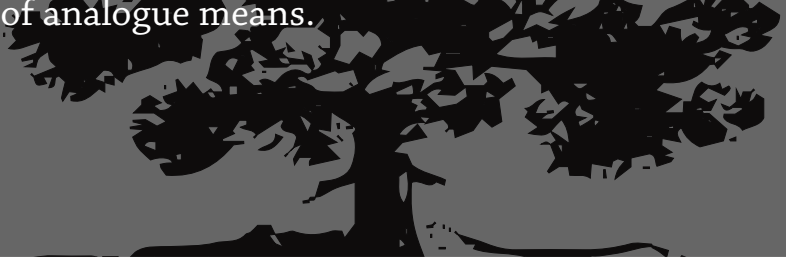




\section{Purpose}

Prototyping new material systems through pavilion-like constructions is an established practice executed by research institutions globally. For example, the Institute for Computational Design at the University of Stuttgart has produced a pavilion each year (est. 2010) to apply research in computational design, fabrication, and material applications. Building at full-scale has the capacity to reveal new knowledge, such as that obtained while fabricating the initial beam prototype (Part I: Branches). Working at the scale of a pavilion will demand heightened consideration for connections, foundations and cladding using the established computational methods.

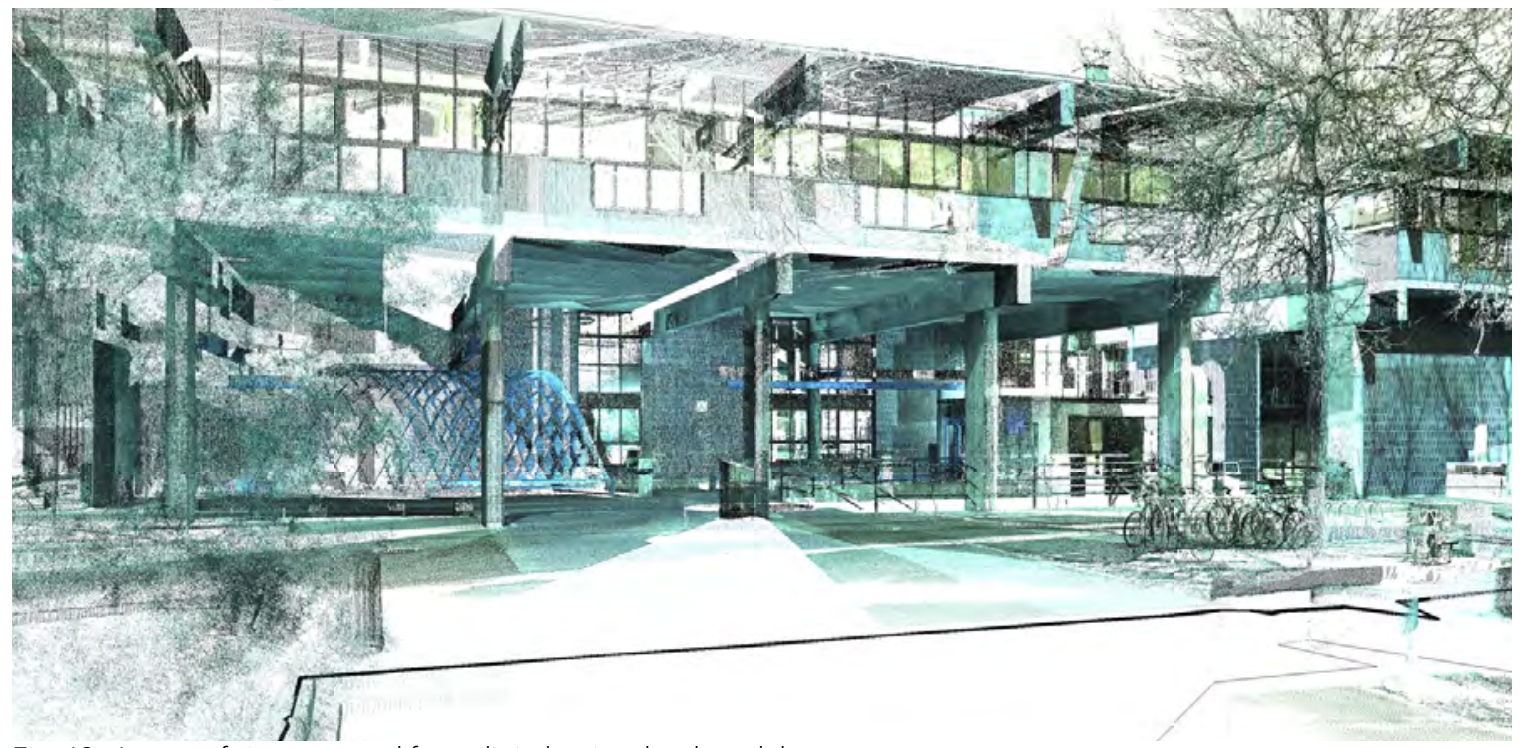

Fig. 19 - Image of site extracted from digital point cloud model

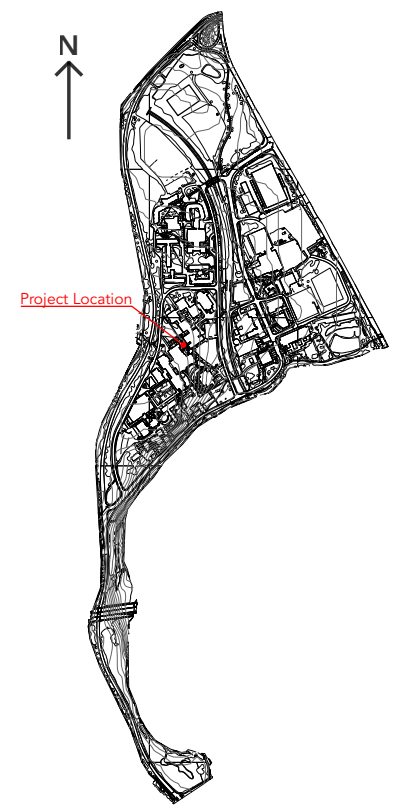

Site Selection

Because the nature of this pavilion is intended to explore computational methods the selection of a site located on campus close to the fabrication facilities was preferred. The site selected is located immediately outside the front entrance of the Azrieli School of Architecture and Urbanism and will integrate with the existing concrete steps and accompany concrete landscape features.

Fig. 20 - Campus context plan with project location 


\section{Analysis of Immediate Context}

As described in the encompassing essay of this thesis, Part III: Trees, an objective, analytical, computational methodology was employed to extract various physical and environmental aspects of the site. These environmental factors would be utilized to generate a basic shell form for the pavilion. It is important to stress this process was not deterministic but inquisitive in nature, seeking only to be informed by the site. In order for this process to begin, a digital point cloud of the university campus, produced and provided for this thesis by Prof. Steve Fai of the Carleton Immersive Media Studio, was obtained (fig. 30). With this highly accurate digital representation of the built environment, the context of the site could be modeled with precision.

Aspects of the point cloud model, such as topography, existing buildings, landscape features and vegetation masses were incorporated into an analytic model. The following analyses were conducted with environmental analysis components for Grasshopper3D; solar radiation analysis, wind, rainfall runoff, and glare from the glazing of adjacent buildings. It

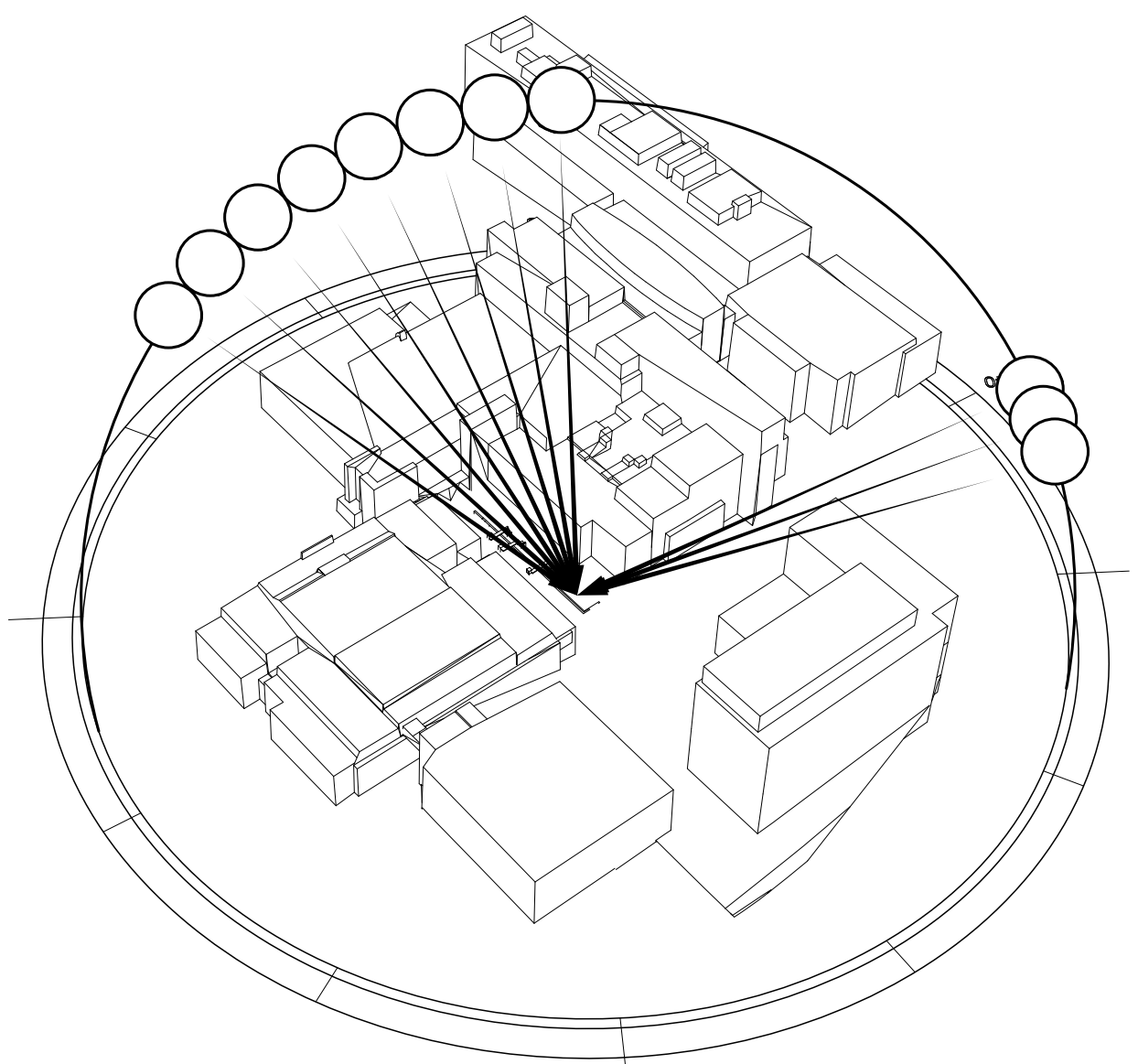

Fig. 21 - Instances of direct solar exposure to the project site 


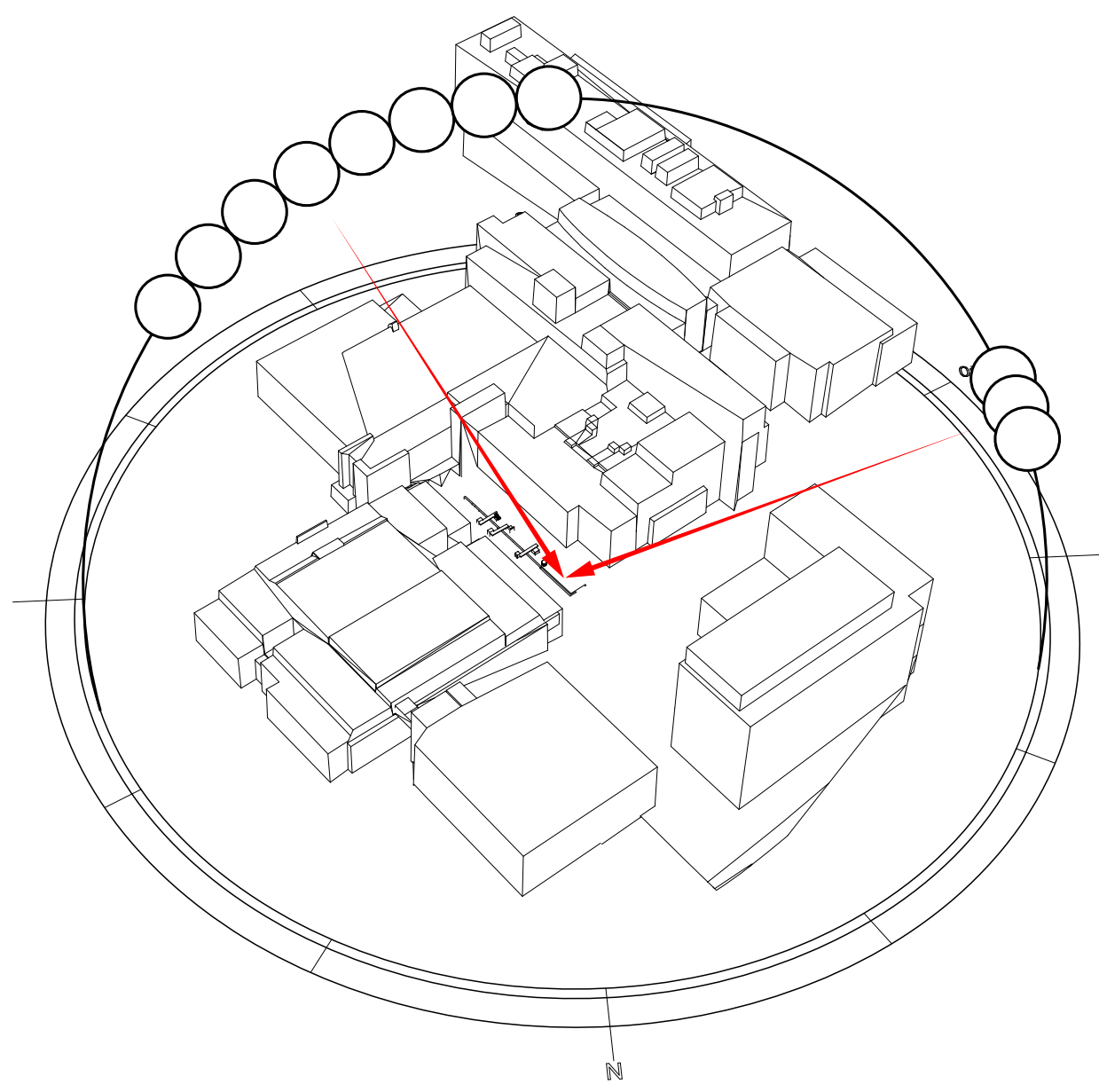

Fig. 22 - Average of all solar vectors exposed to the project site

became evident that of the analyses conducted few would have a generative effect on the pavilion form. This pavilion, lacking architectural program or building envelope requirements, is intended to serve as an experiment in which the fabrication methodologies developed can be further tested. During the analysis however, it was discovered that the site only receives direct sunlight during two specific times throughout the day. The restrictive nature of the solar potential for the site served as the primary form-generating force.

After a specific day of the year was selected, April $21^{\text {st }} 2016$, the periods that would receive direct sunlight were defined - a longer period during the morning and a short period in the afternoon. The solar vectors during these times were calculated and documented so they could be used in the form-generating algorithm. Once again, it is imperative to stress there was no preconceived notion defining the form of this pavilion. The solar vectors collected from this first site analysis exercise were transferred to a form-generating script. 


\section{Form Generation}

What are the implications of architectural form that is the result of computing a series of environmental factors? Does this have any effect on the quality of the space created? The relevance and integrity of creating form with this method is that formal aspects of this pavilion actually respond to the immediate environment and their formal attributes can be quantified and controlled. The purpose of form generation for this thesis is to have a computed surface that is the result of information-based analysis that can be further utilized with the Computational Grain Realignment methods.

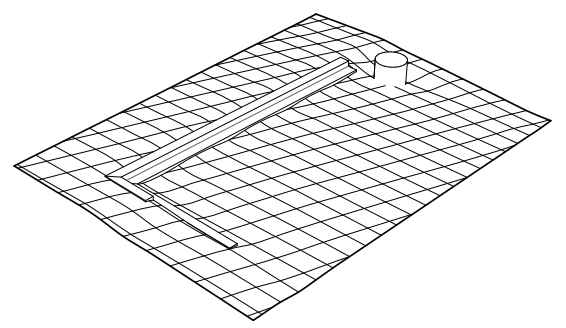

Fig. 23 - Initial analysis condition: A concrete step formed in the landscape next to a cylindrical concrete pier of no specific function

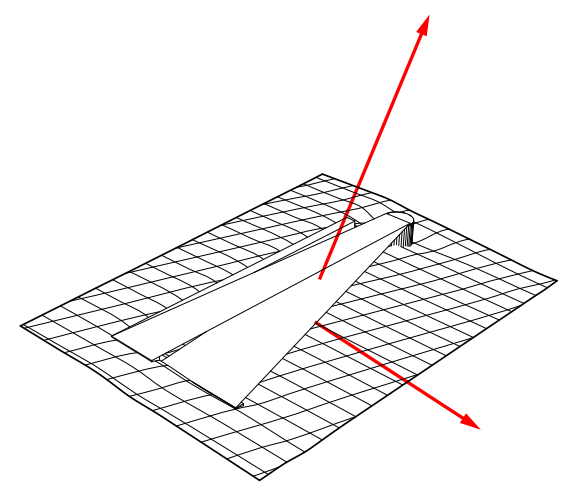

Fig. 24 - Average of solar vectors: (Fig. 25) are implemented as physical forces to "pull" the planar surfaces depicted in the directions shown. The surfaces are fixed at their ends

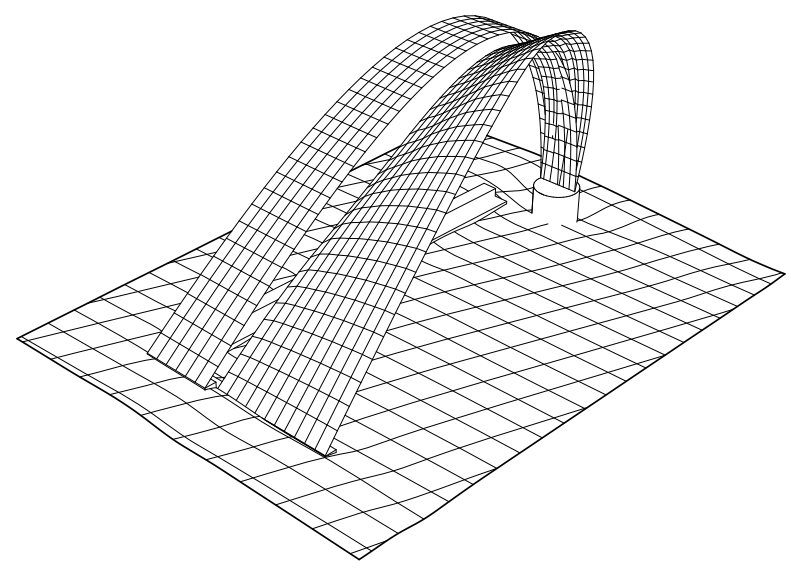

Fig. 25 - The resulting surfaces arch over the concrete steps in a variety of fully manipulatable configurations 
To generate the pavilion form using the acquired solar information, a mathematical average of the solar vectors entering the site would serve as the generative. The analysis was completed by the Kangaroo physics engine, a component for the Grasshopper3D visual scripting interface. A series of four points were placed on the site that would serve to define a starting footprint for the pavilion. The 4 points were developed into a flat planar surface situated on the ground, with no habitable volume. The solar vectors were then utilized as directional forces that "pull" the flat planar surface in the direction of direct sunlight, creating an arch-like shell form.
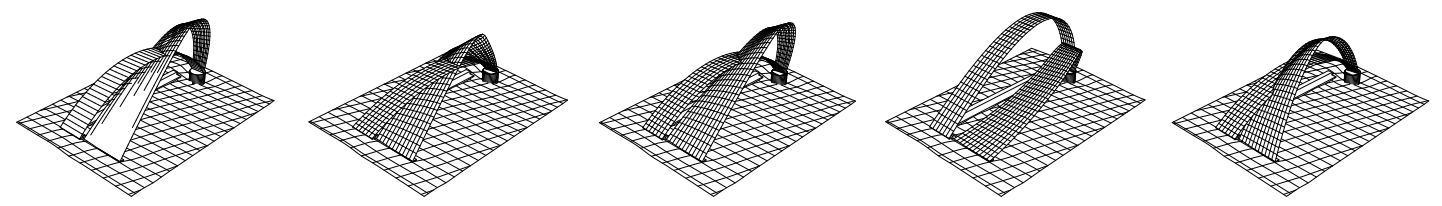

Fig. 26 - Schematic diagram of utility script used to sort through available principle stress values and return a domain of the highest value. This computational logic was developed and implemented to retrieve the highest principle stress values, in both tension and compression, that would be used to fabricate the digital beam prototype.

The multi-parameter nature of this method meant that an infinite number of formal configurations were possible and that certain configurations would produce a form that was "superior" to others. To remove aesthetics preferences from determining the final form, an optimization algorithm was generated to find an ideal form, or range of ideal forms that could be generated. To do this, an optimization target was required and total deflection of the shell form was selected as the structural attribute to optimize. The evolutionary solver and finite element analysis in Grasshopper3D were utilized to compute the possible configurations that would produce a shell form. The total deflection of the shell was measured with each successive configuration. The resulting shell form was superior to the other possible shell forms because it would inherently deflect less than the other possible forms, making it the preferred candidate to further develop with the grain realignment method.

\section{Form Analysis}

The geometry analyzed in developing the digital beam prototype was planar and did not possess habitable spatial qualities. In order to explore spatial and architectural possibilities, the
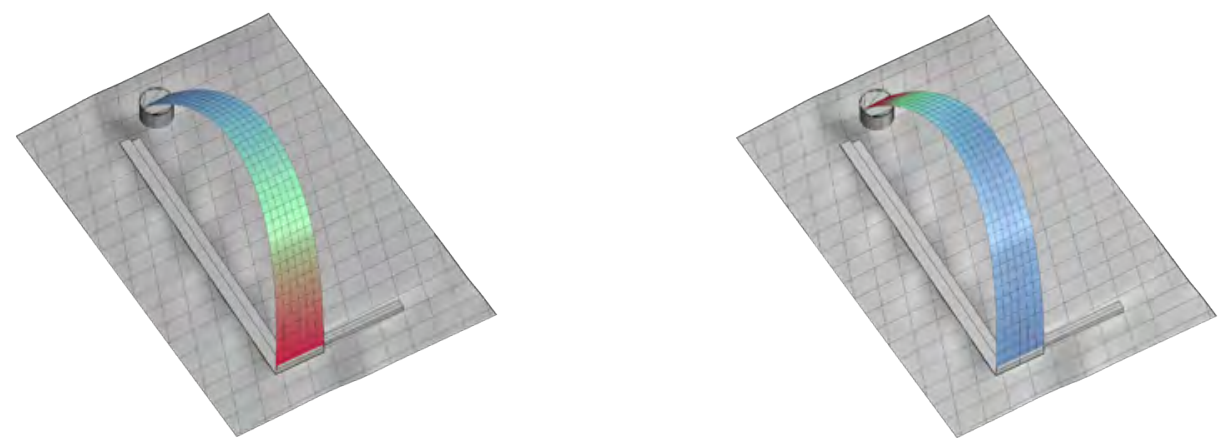

Fig. 27 - Initial shell stress simulation results 


$$
\begin{aligned}
& 111 \\
& 110
\end{aligned}
$$


investigation shifted from individual structural components to space-defining geometry that could serve as architectural space. For the purposes of this investigation, it was determined that three-dimensional shell geometry would be explored. Given the complex nature of forces acting on and within a shell, this transition from 2D planar to 3D shell geometry meant the analytical method utilized in chapter one would need to evolve.

Shell geometry is more complex than a planar surface because it has thickness and the local orientation of the shell relative to the force applied is constantly changing. To extract reliable data that would accurately describe the principle stresses acting within the shell the structural analysis was conducted at three critical locations within the thickness of the shell;

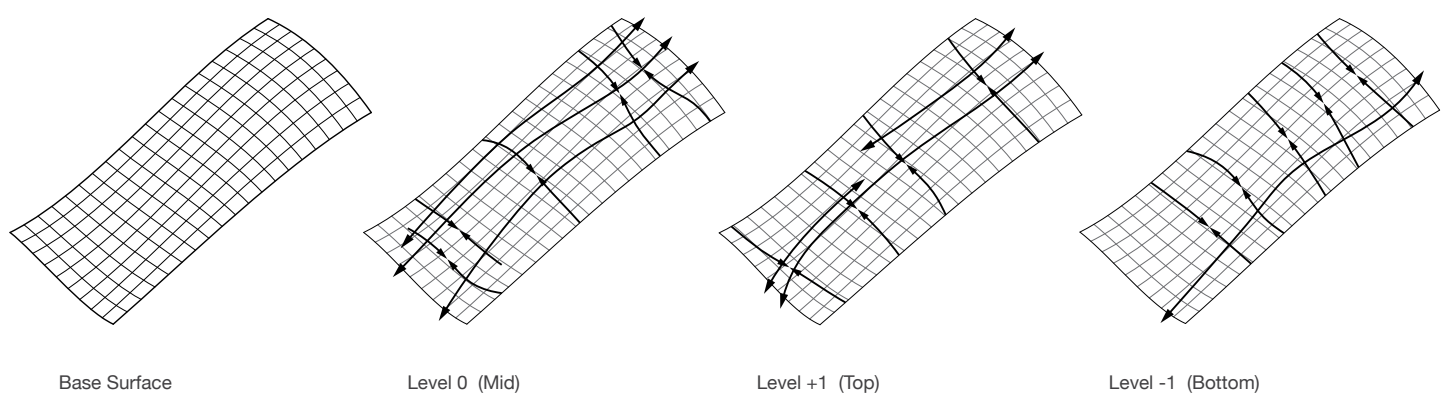

Fig. 30 - Principle stress trajectories shown at various locations within shell thickness

its bottom, middle, and top. The principle stresses vary depending on their location within the thickness of the shell. The analysis generated a network of curve geometry indicative of the principle stresses acting at various shell locations. These curves would inform the design of the required structural members and would be required to develop the shell surface with the Grain Realignment method.

The image sequence depicted (left/right) illustrates the initial stage of the following analysis in which shell form is analyzed with the finite elements method. Using the same method in Part I, the geometry that is of interest for development and fabrication are the principle stress curves acting through the surface. Since developing the beam prototype in Part I the performative nature of the script has been improved so that it returns principle stress curves for three locations within the thickness of the shell surface. The resulting curves will serve as the generative geometry required to begin fabricating structural components of the pavilion.

\section{Pavilion Fabrication}

Unlike the digital beam prototype, the shell form generated for the pavilion revealed new geometric challenges to be addressed with a revised fabrication strategy. The digital beam required the veneer to bend and conform in one direction only, which is possible within the 
material limits of the veneer. The pavilion shell has surface curvature in two directions, meaning that a planar layering strategy similar to that used for the digital beam would not be sufficient. Before committing to full-scale pavilion construction with an undetermined fabrication strategy, it was decided that a series of material studies be conducted that would inform the fabrication methods. These studies were conducted to determine the geometric limits of fabricating three-dimensional, doubly-curved forms using multi-ply laminations of flat, planar layers. Of the fabrication strategies explored, a successful prototype emerged as part of a design for the legs of a chair.

The chair legs have geometric characteristics similar to the structural members required of the pavilion - planar surfaces with thickness that curve in two directions. The system of lamination jigs used for the digital beam prototype would not work for these elements given their size, irregularity, and the geometric constraints of the digital fabrication technology available (i.e. maximum cut depth, bed size restrictions, clamping limitations, etc...). Instead, an experimental lamination system was developed that utilized a series of vertical ribs to support the multiple layers at critical, form-defining locations during lamination. Key to the success of this process is prefabricating each unique ply with the $\mathrm{CNC}$ router prior to lamination. This step ensures that once placed within the lamination jig, and in the proper ply order, the multiple plys will assume the desired form as clamping pressure is applied. This lamination method proved successful and resulted in doubly-curved chair legs. This experimentation and resulting chair prototype ensured that the subsequent efforts put forth in developing the 1:10 construction model would hold promise.

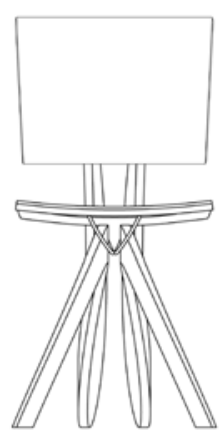

Front Elevation N.T.S.

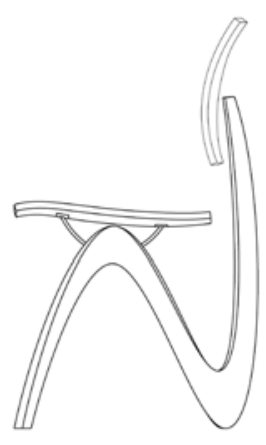

Side Elevation N.T.S.

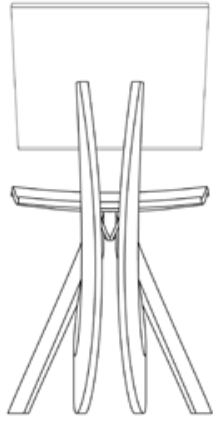

Rear Elevation N.T.S. 


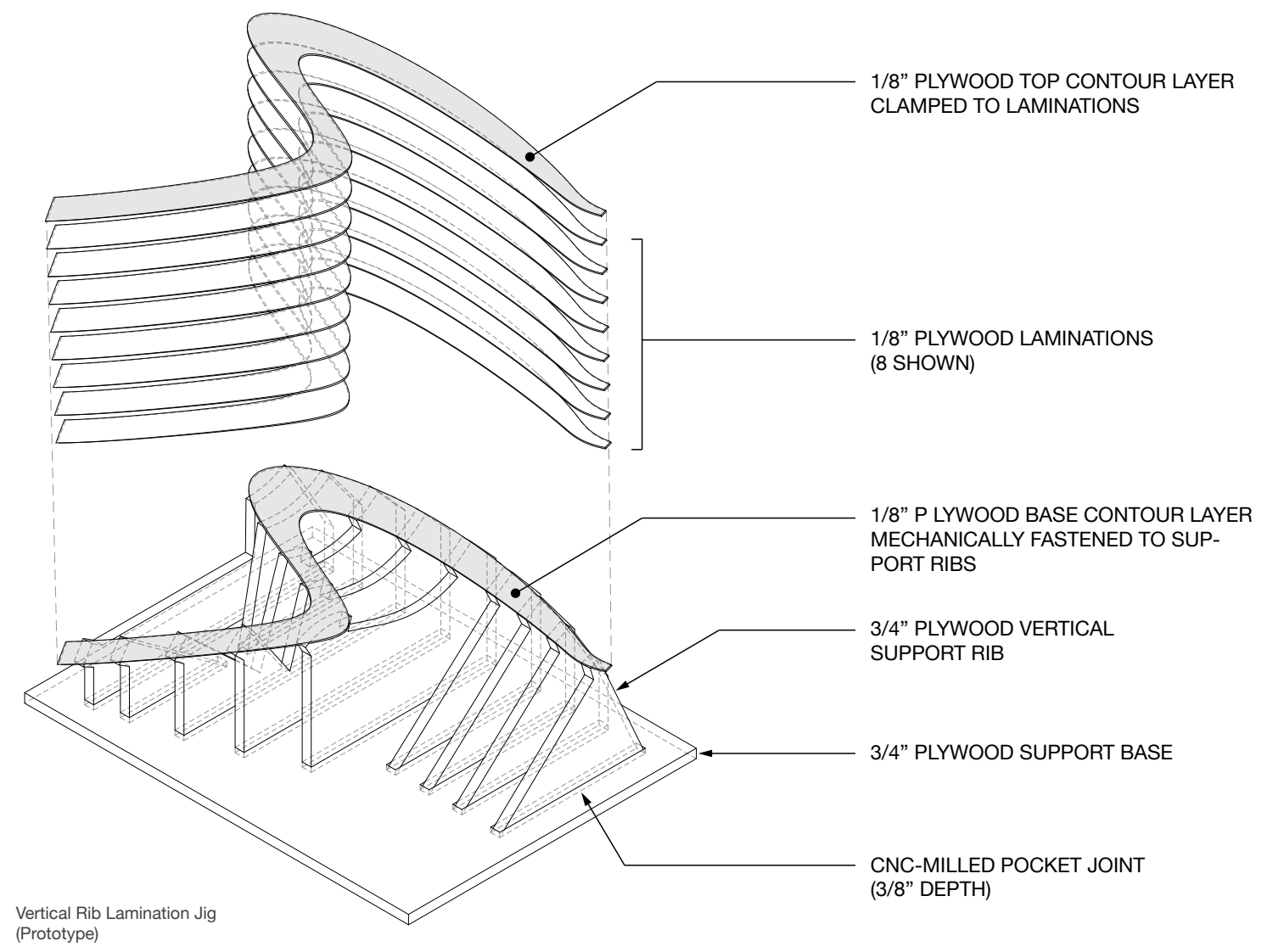




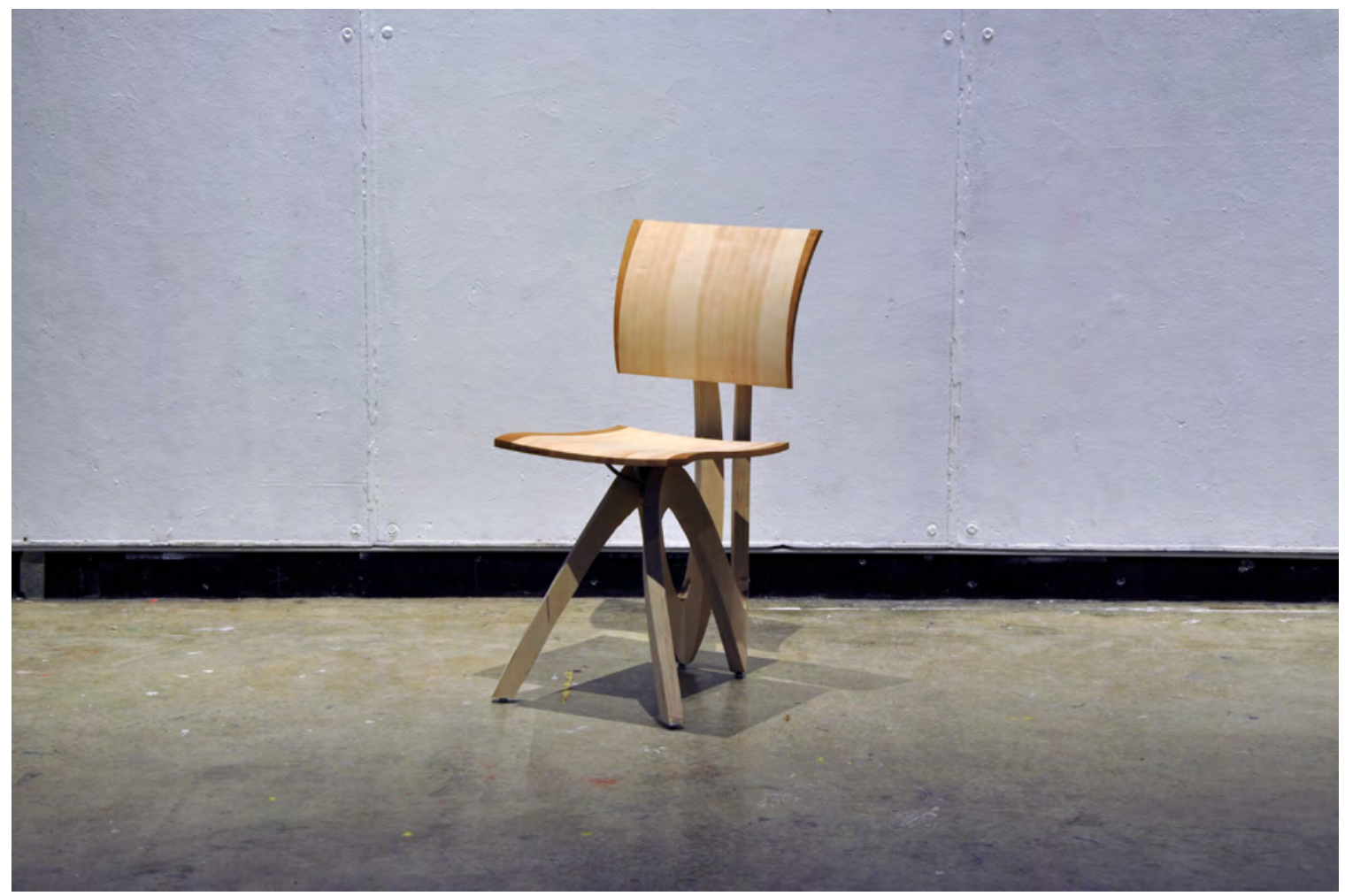

Chair Prototype - 01

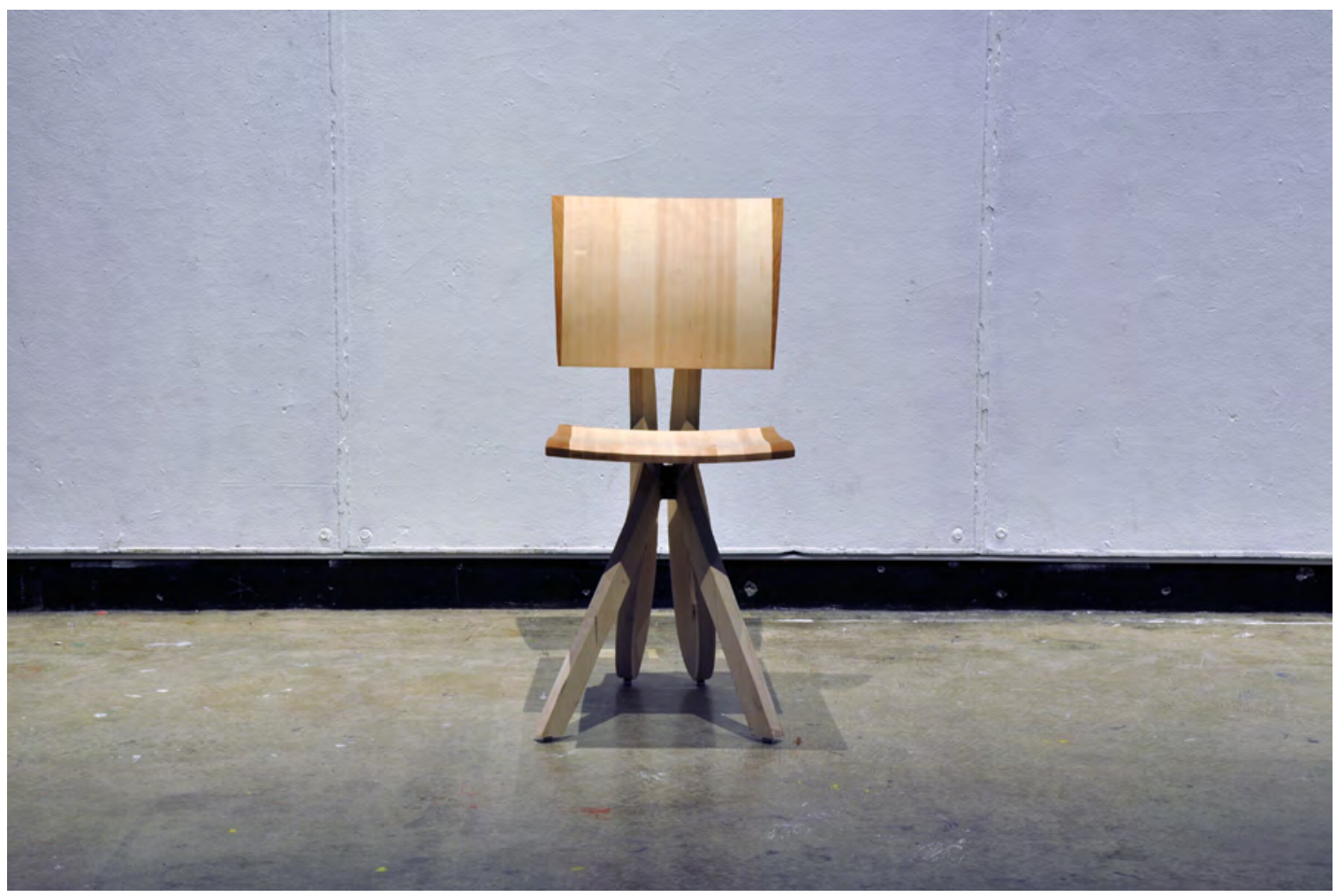




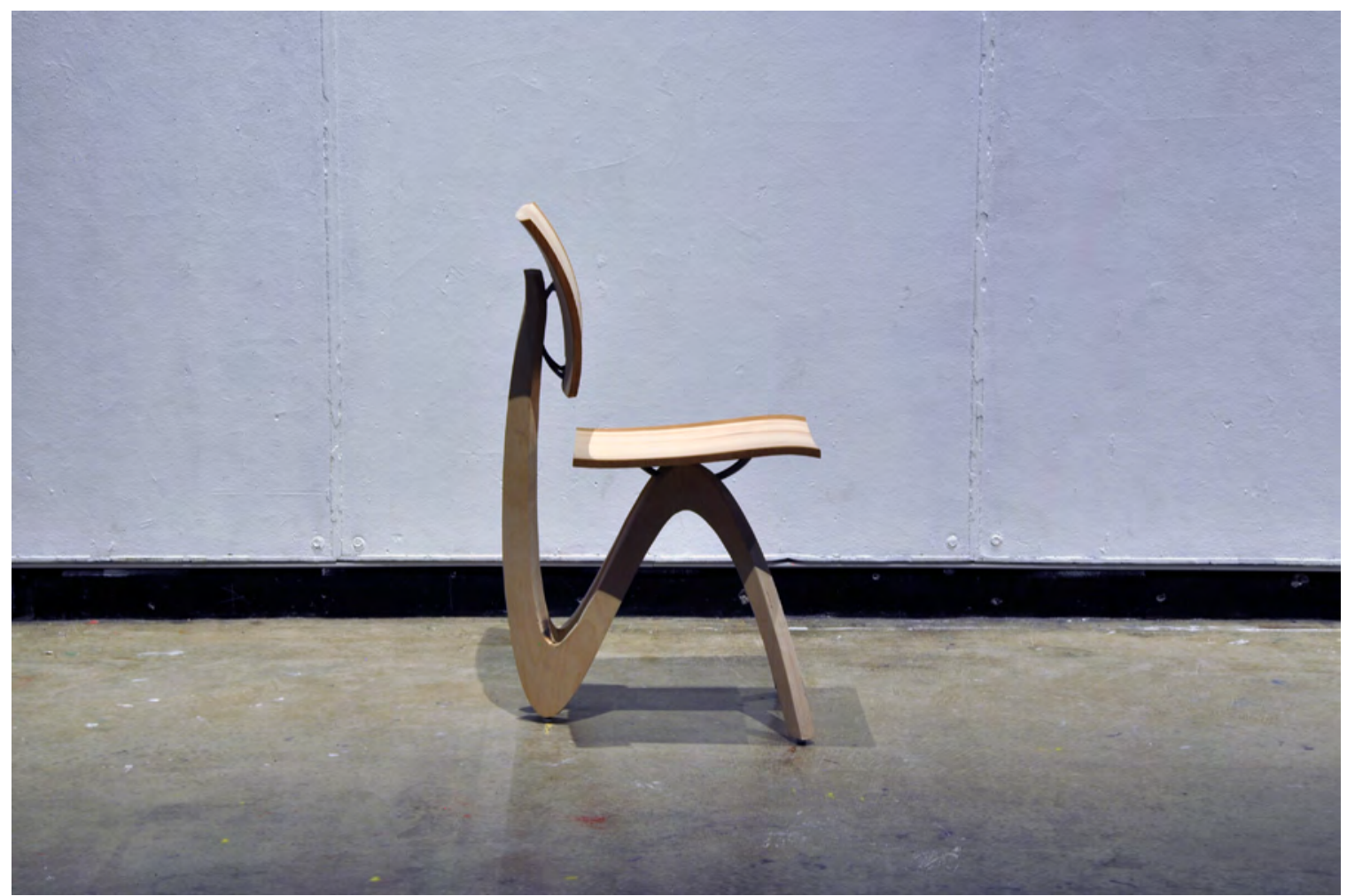

Chair Prototype - 03

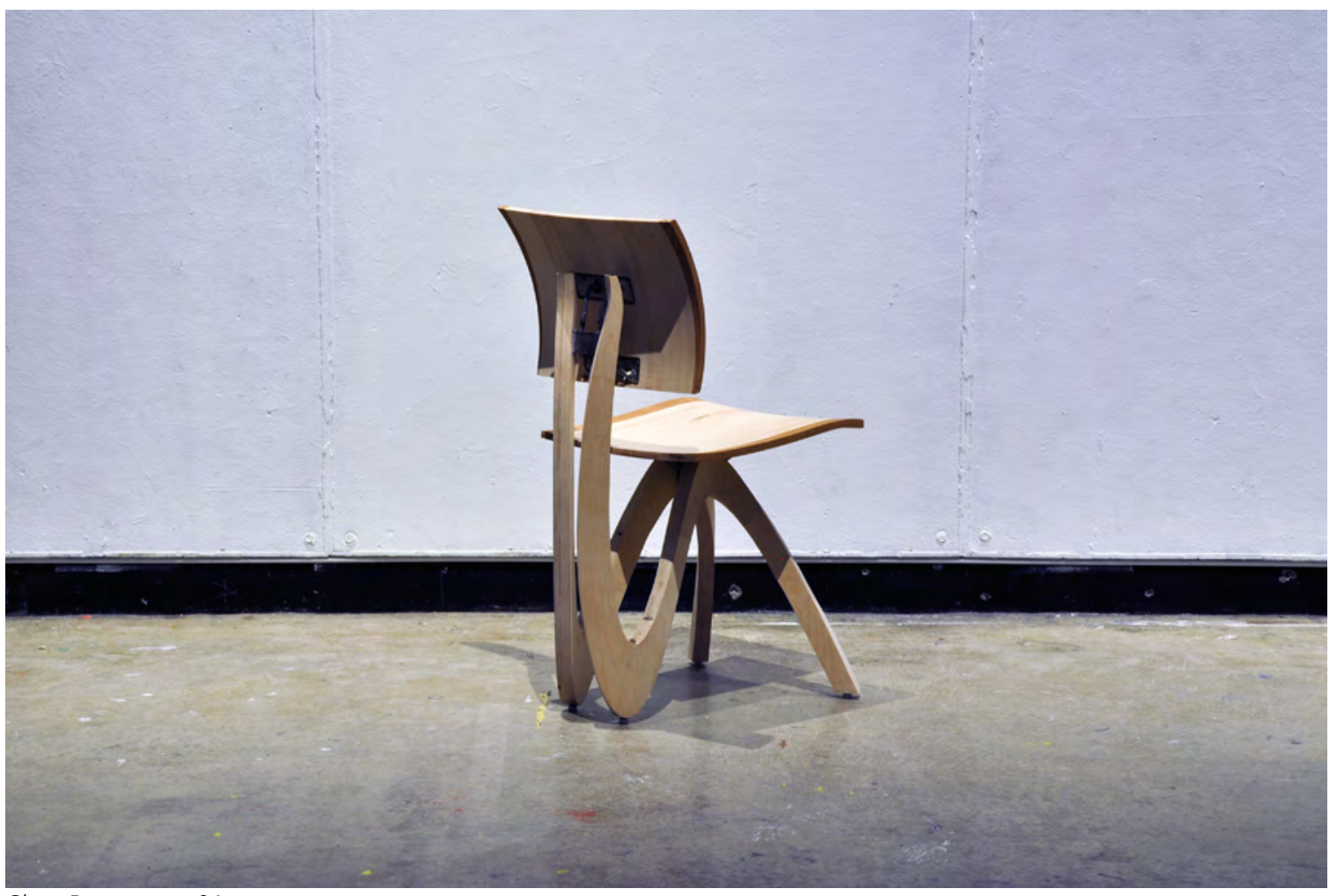




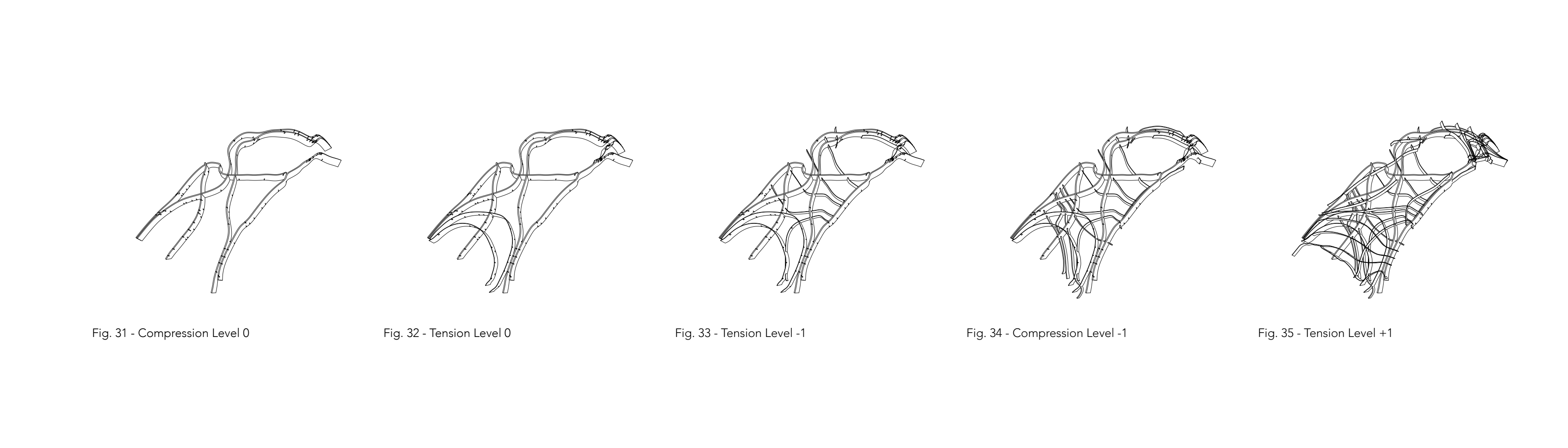


To efficiently process the geometry of the proposed structural members of the pavilion, an additional utility script was developed. This script analyzed three-dimensional objects and generated flat two-dimensional curves that would be used to either laser-cut or $\mathrm{CNC}$ mill the plys for the structural members of the pavilion. Having experimented with both $\mathrm{CNC}$ milling and laser cutting for the production of these members, it became clear that laser cutting is superior to $\mathrm{CNC}$ milling for this specific application because it can achieve intricate details required of the intersecting lap joints. The intricacy of detail that a CNC machine is capable of is limited to the scale of the object being milled and the radius of the cutting bit. For example, an interior corner will always have the radius of the cutting head - a $90^{\circ}$ interior corner is not possible and if this is not taken into consideration early the joinery will not be achieved as designed.

As an exercise in advanced digital fabrication strategies, constructing a detailed model revealed elements of the fabrication process that were successful and those that were not. The geometric limitations of the veneer were revealed immediately and curvature benchmarks were established that could later be used to inform the design algorithm.

\section{Post-Fabrication Analysis}

While the 1:10 construction model was successful overall, several critical flaws were revealed that will be addressed here which could be used to inform a revised model. The areas for improvement include modifications to the computational process that generated the geometry as well as alternative material choices and fabrication technology.

The utility script written to process the FEA data was written to search the complete list of principle stress geometry for the highest values in a given range. It does not account for the nature of the curve geometry and what resulted in several instances were tight curvatures that could not be achieved with the wood veneer. If revised, the utility script would first filter all available principle stress curves to those with a median curvature value that is within the elastic range of the wood veneer. Only then, would the script search for the highest principle stress curvature. This would ensure that the resulting geometry is possible to fabricate using the selected material and could be modified if there is a change in material.

An effort was made to develop a new lamination jig system for the purposes of fabricating the structural members of the pavilion. The chair prototype successfully demonstrated methodology which was used to laminate several critical structural members in the pavilion. However, while building the model it became clear that separate lamination 


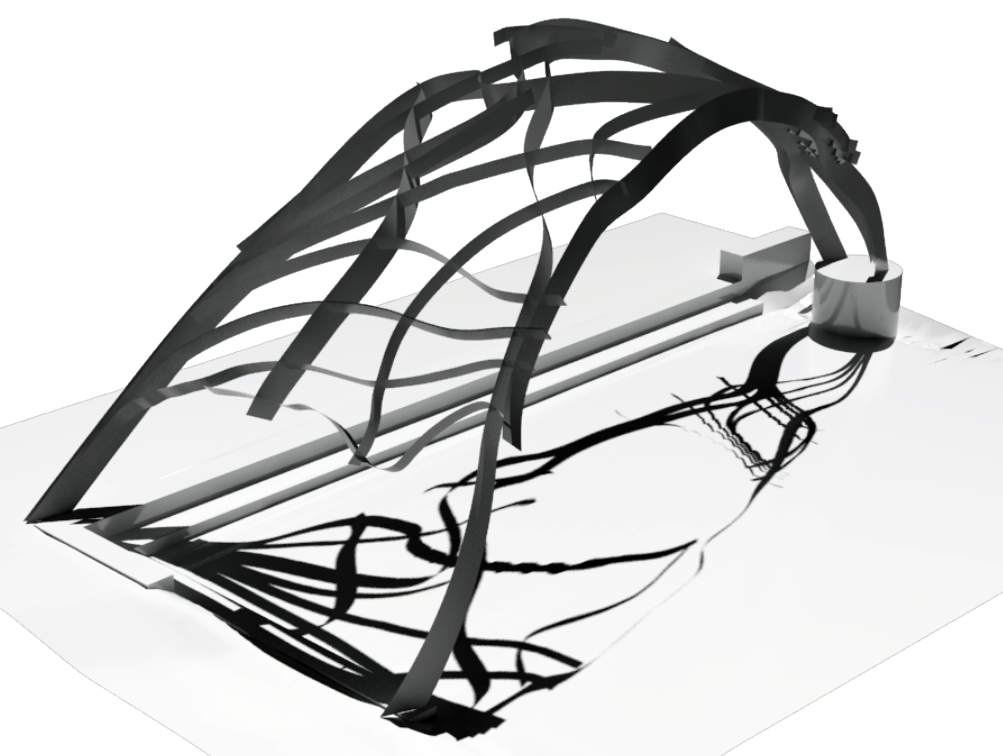

Fig. 36 - Final pavilion form to be fabricated (Northwest perspective)

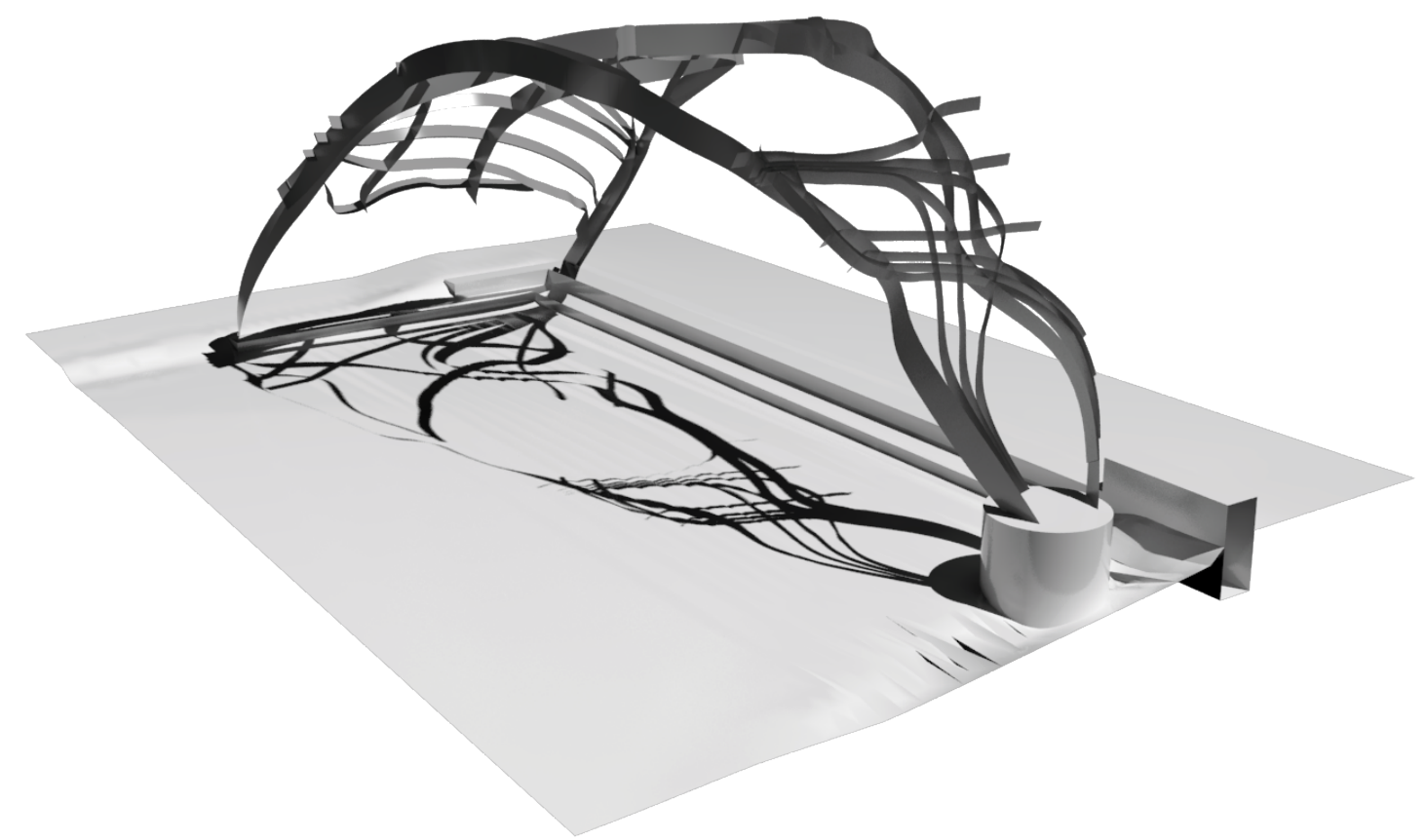

Fig. 37 - Final pavilion form to be fabricated (southwest perspective) 


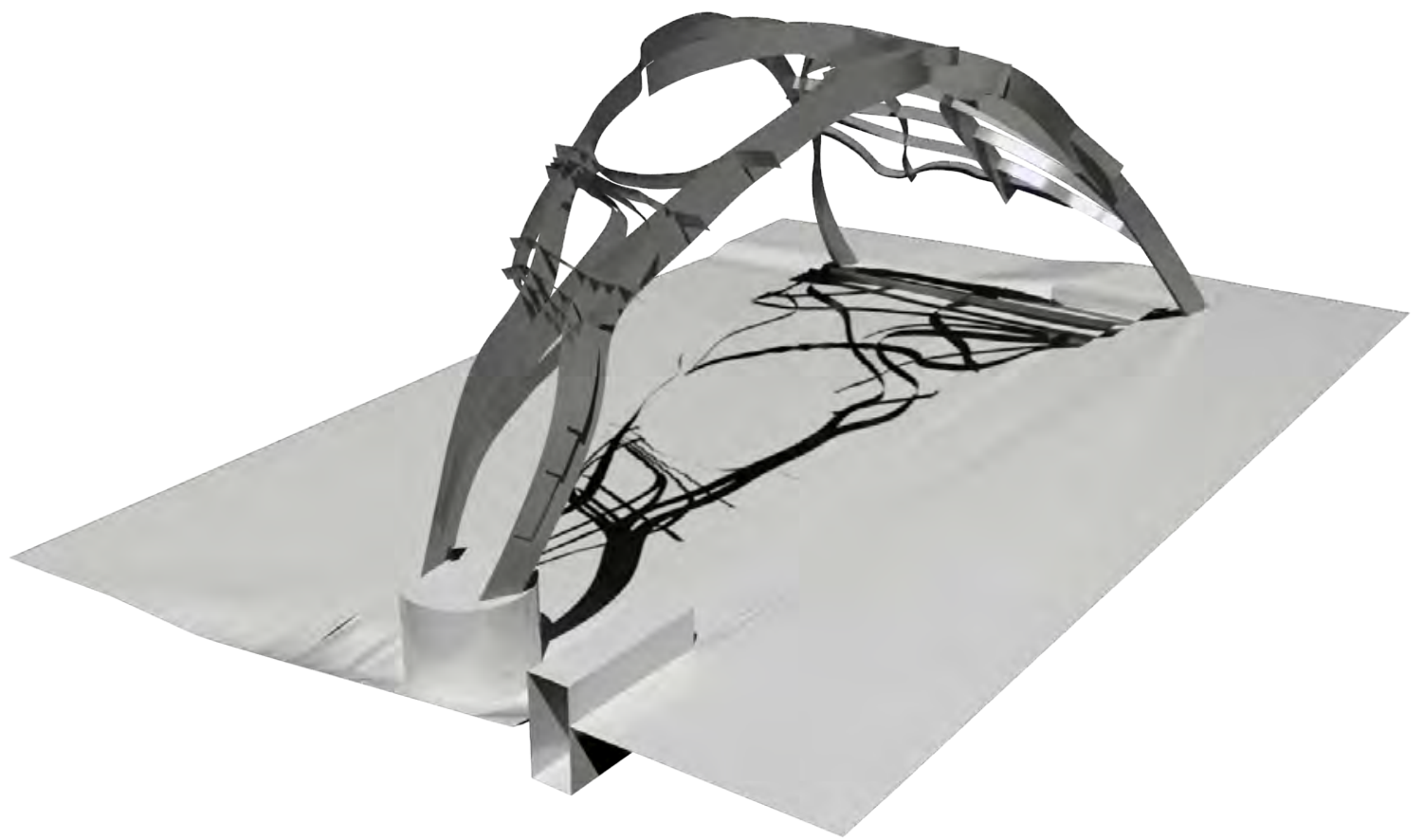

Fig. 38 - Final pavilion form to be fabricated (Southeast perspective)

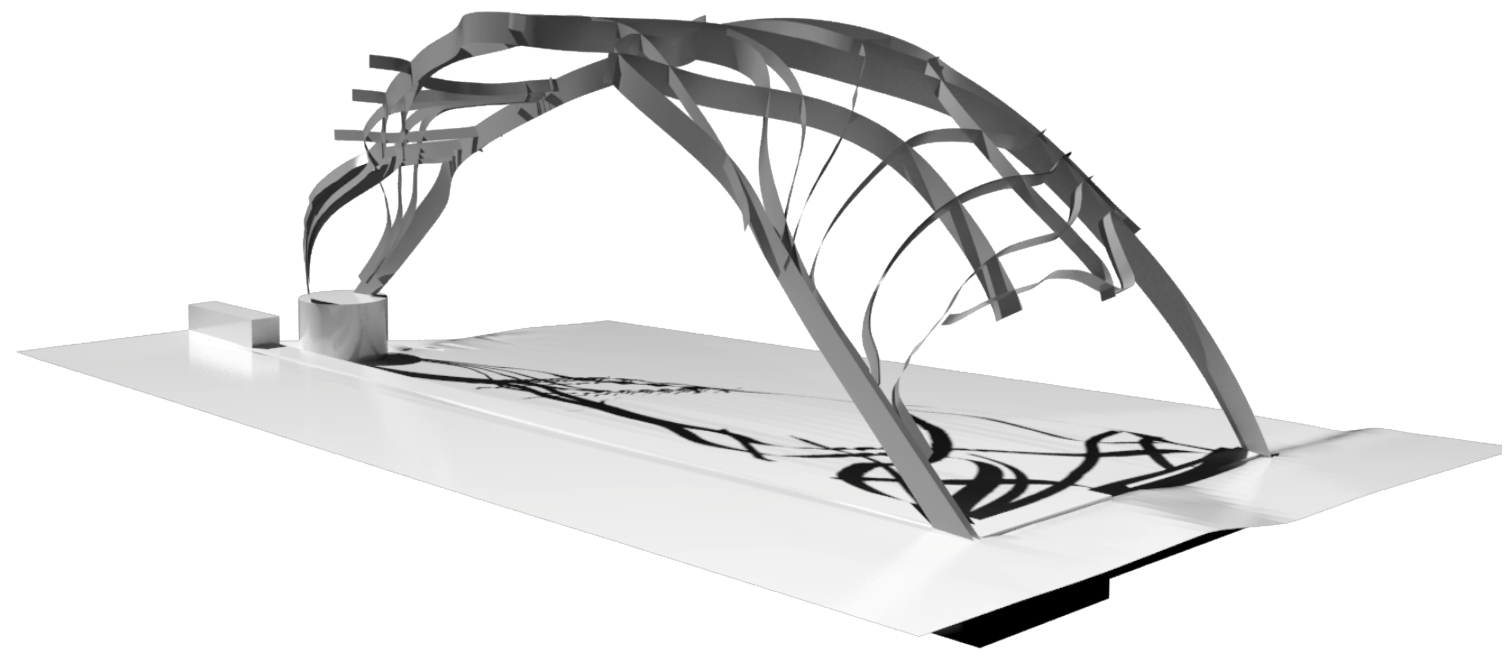

Fig. 39 - Final pavilion form to be fabricated (Northeast perspective) 


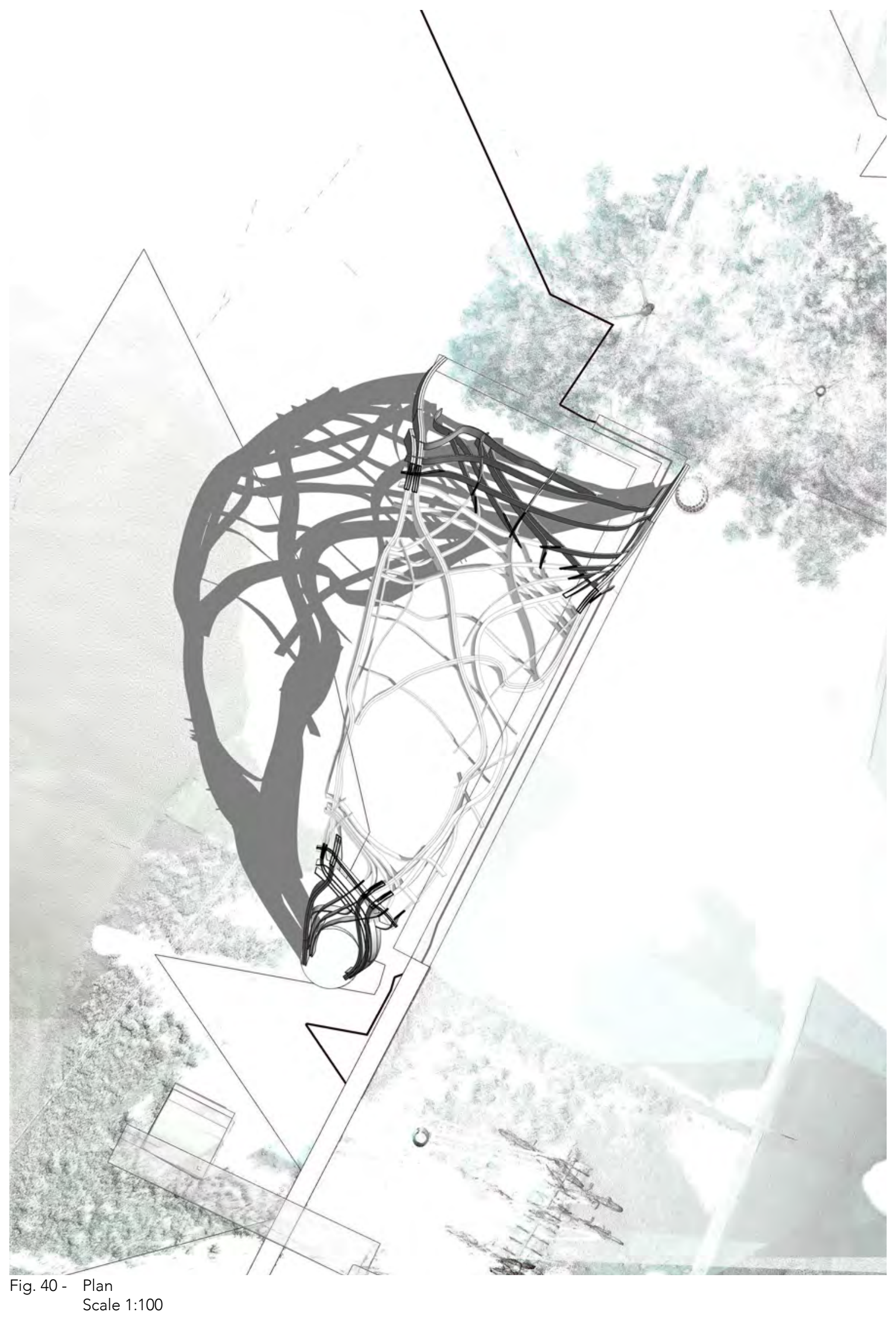




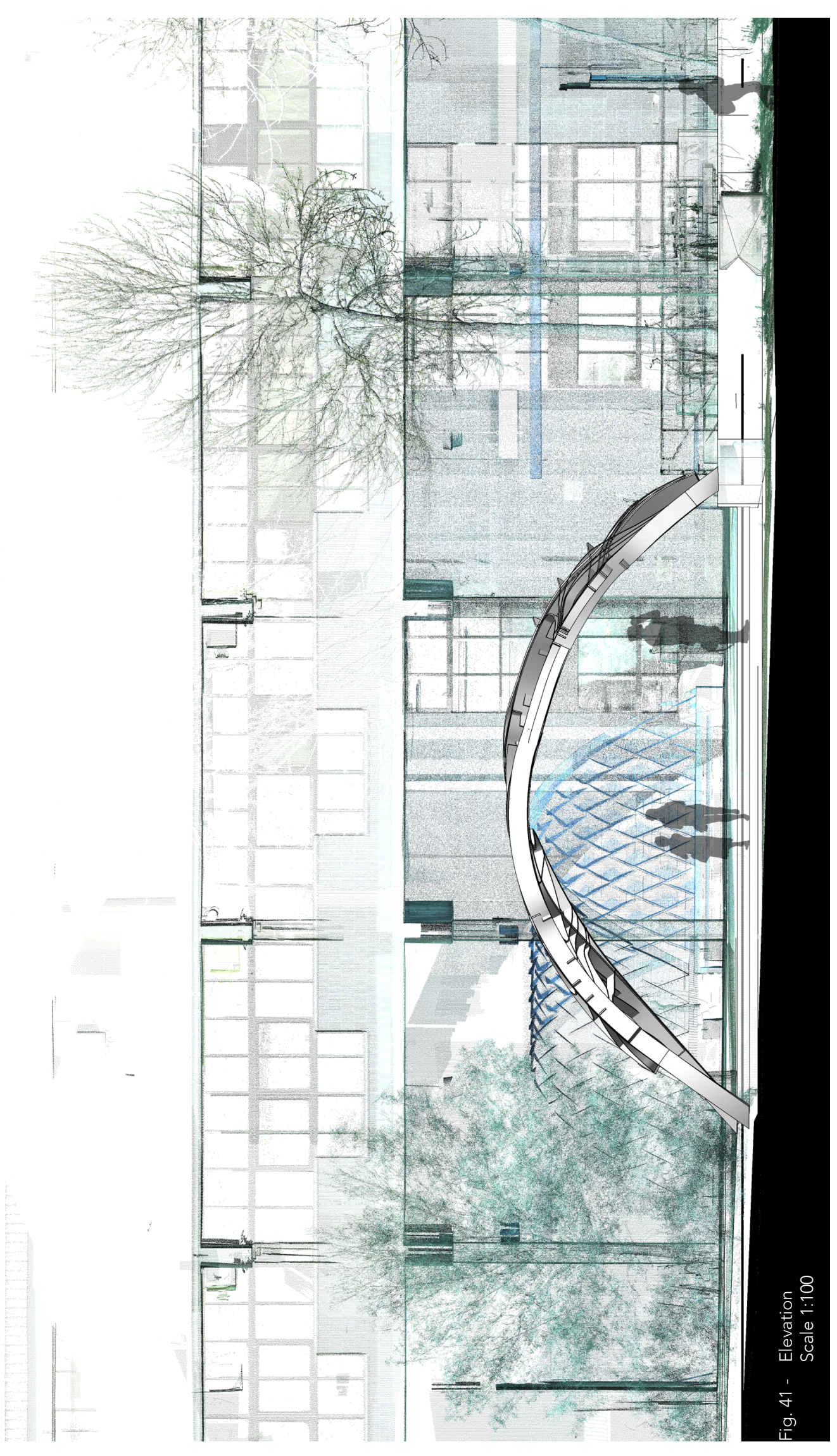




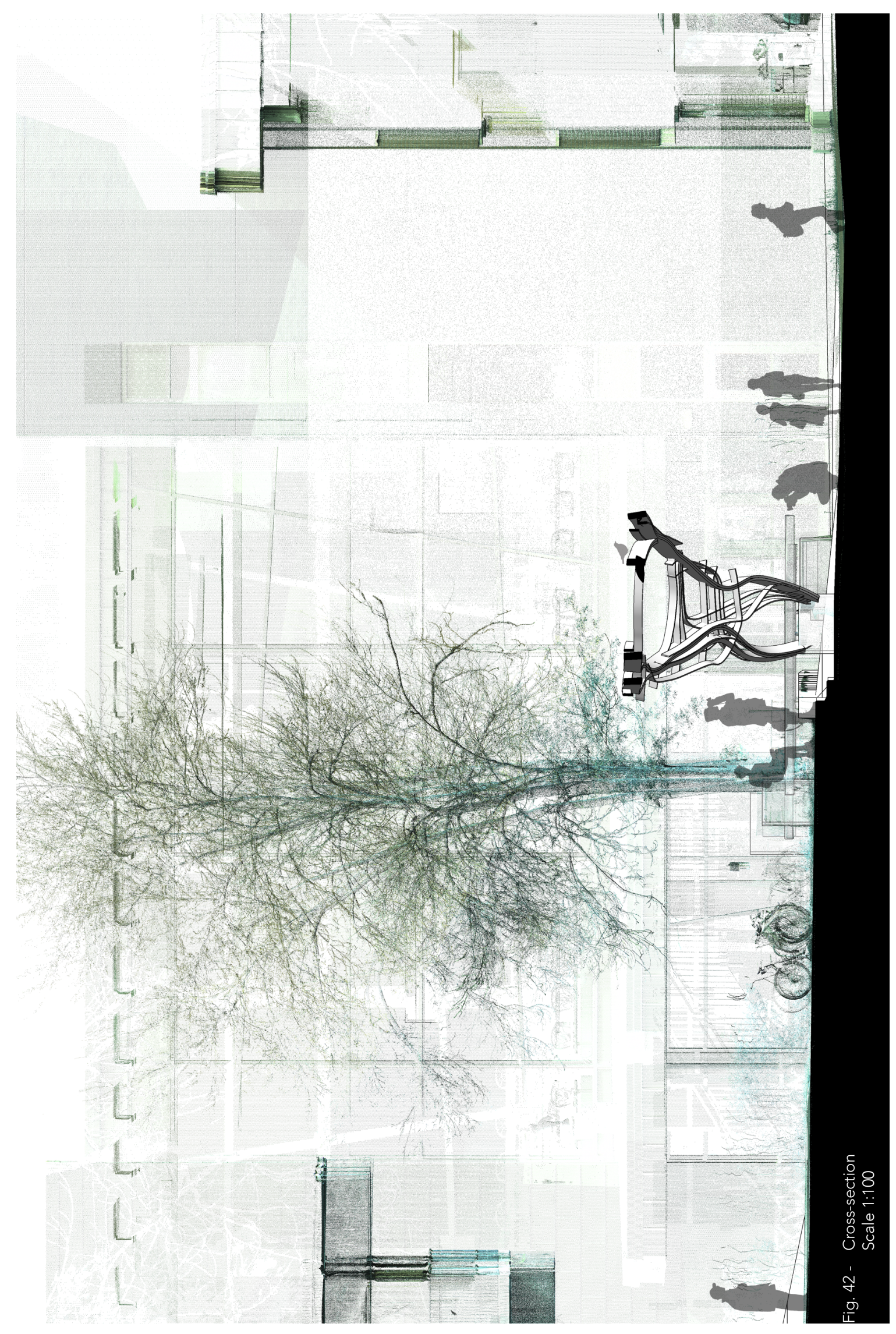




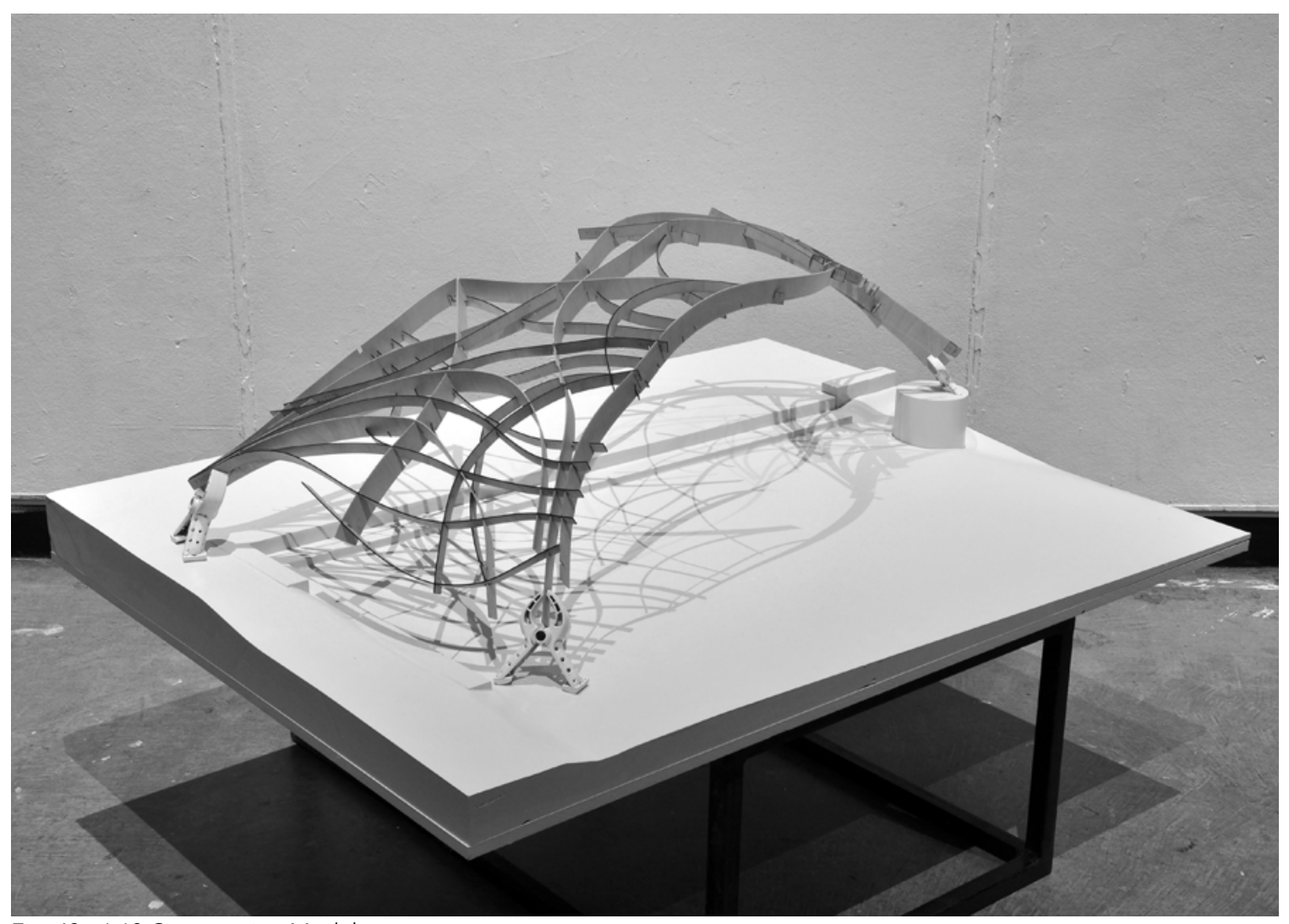

Fig. 43 - 1:10 Construction Model

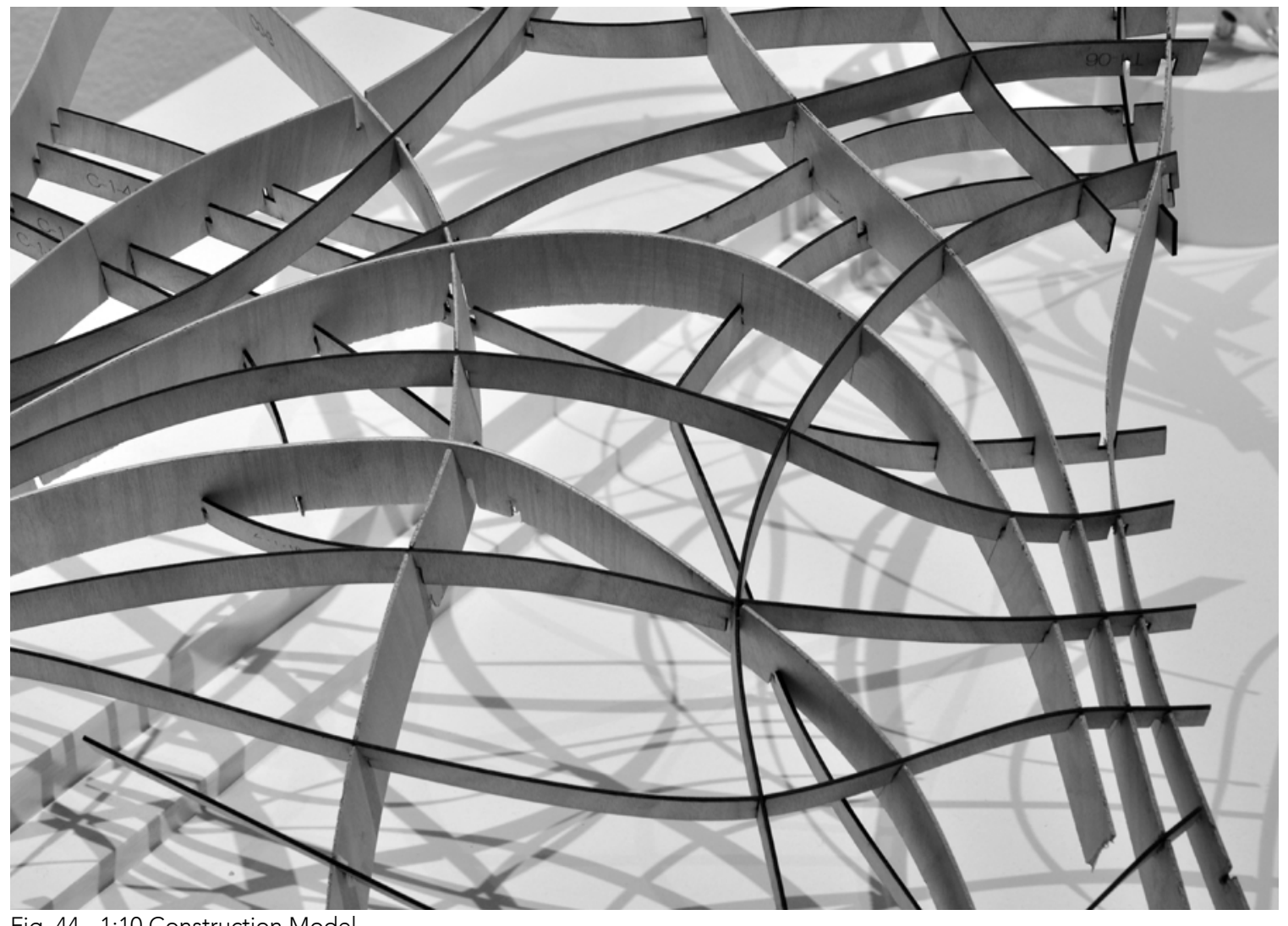

Fig. 44 - 1:10 Construction Model 


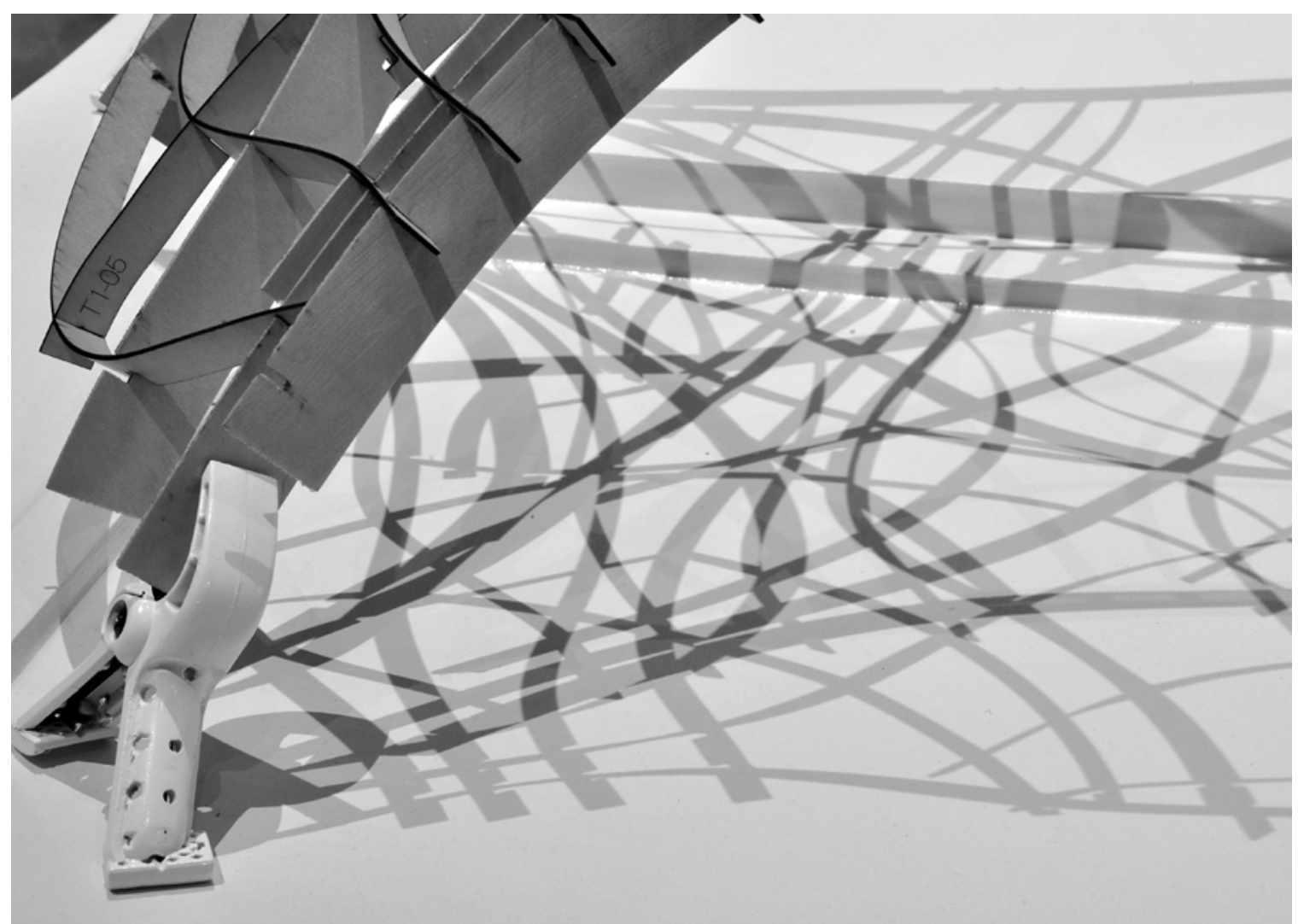

Fig. 45 - 1:10 Construction Model

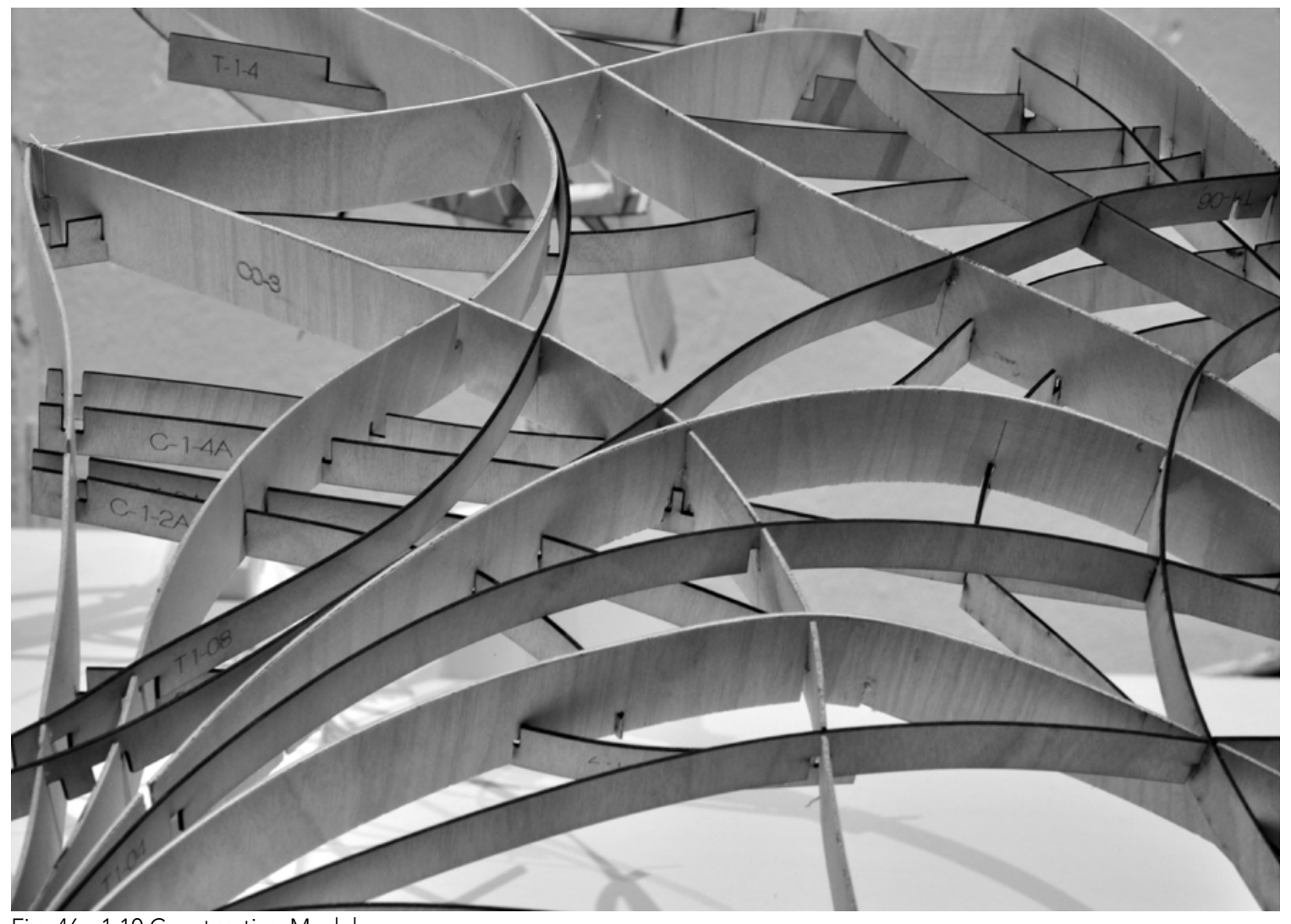

Fig. 46 - 1:10 Construction Model 
Conclusion 


\section{Conclusion}

Digital Wood represents a marriage of three components: computational design, digital fabrication, and material science. The goal of this thesis was never to develop new materials, but instead to use technology to develop new ways of analyzing, working with, and applying existing materials to spatial conditions. Structural timber is experiencing a renaissance in the $21^{\text {st }}$ century as architects and engineers renew their interest in this material system and look for ways to advance wood construction systems. Increased demand for low-carbon content materials that are renewable and energy efficient has shifted focus from the tried staples of steel and concrete for large building construction. Many architects are now looking to wood and structural timber systems for tall building construction. Precedents around the globe have demonstrated that structural timber is a suitable, if not superior alternative to conventional construction methods.

The term mass timber has become commonplace when discussing tall building construction. This new material system has demonstrated that tall buildings are possible without steel and concrete. However, wood-steel and wood-concrete hybrids are showcasing methods of construction that strategically employ each material in a manner that best utilizes its mechanical properties. Further innovations in material applications such as these will be required to meet the social and environmental demands that will surely result as the world's population continues to urbanize. Global population increase coupled with rapid urbanization will require buildings to be constructed in ways that employ lasting, renewable and energy-efficient materials that will ease the burden in the planet. This project strives to make a meaningful and lasting contribution to the field knowledge of structural timber by demonstrating alternative applications for wood products through the use of computation and digital fabrication technology.

Digital Wood: Computational Grain Realignment has explored a very specific area of research that will aid in the advancement of structural timber building products. This thesis holds that the very best construction system can only be achieved when material properties are considered first and subsequently used to inform the design and the fabrication of the system. The concept for this project was inspired by existing research into the mechanical behavior of the tree carried out by Claus Mattheck. The tree grows and develops as a result of the external loads it is experiencing and develops grain and fibre orientations to uniformly distributes stress. The concept of a structure developing as a direct response to the load applied served as the guiding principle while developing the beam prototype detailed in the first chapter. The complexity of the natural system that inspired this working structural prototype could not be achieved without computation.

An immense effort was put forth developing the computational methods necessary to analyze and subsequently fabricate the digital beam prototype. A combination of several 
software applications was required to model, simulate, analyze and then process data for fabrication. This resulted in a computational method that can be adapted to a variety of structural conditions where the highest principle stress values are sought. This has direct applications to structural timber design, as this project demonstrates, but can be applied to structural conditions that utilize other material systems. Additionally, this process yielded several useful algorithms, referred to as utility scripts in the text that can be applied to a multitude of scenarios in which large volumes of data must be processed to find specific numerical values and the locations of these values.

Fabricating the beam prototype served as an early performance indicator of the total working methodology that was developed. The merits and applicability of the computational methods would be lessened if an effective fabrication strategy were not developed. Early prototypes were unsuccessful in that the process used in their development would not scale to industry-level fabrication, which had been an objective of this thesis from the early stages. Only after selecting to work with wood veneer was an effective fabrication process developed that produced successful prototypes. Aside from serving as a proof of concept, in that the computational methods developed could in fact yield an object that is possible to fabricate, the beam prototype demonstrated several key principles that align with the renewed interest of structural timber embodies.

The beam prototype utilizes wood in and effective and efficient manner by aligning the principles stresses of a structure to the grain orientation - the most effective grain orientation for load bearing. The process by which the beam prototype was fabricated reduces a significant amount of waste in comparison to the fabrication methods mills have conventionally used. Rotary cut veneer allows for control over grain orientation in fabricating structural elements, but is also the most materially efficient wood product produced because there is almost $100 \%$ utilization. The additive process of laminating thin veneers to develop mass elements significantly reduces waste and produces structural components that can be further articulated through $\mathrm{CNC}$ machining. $\mathrm{CNC}$ machining is a subtractive process in which elements are cut from a larger piece of stock, producing volumes of waste material. This project developed a method of precisely situating objects within the CNC machine bed to be machined, eliminating the need for objects to be cut from larger stock and once again reducing material waste.

The working methodology developed and resulting beam prototype of Chapter I represent the potential for an alternative material system to be adopted by industry. The Digital Wood computational method is capable of powerful data processing in structural simulations. The fabrication methods have demonstrated that existing material types and digital fabrication tools can be appropriated for these applications, given slight modifications to the conventional use of $\mathrm{CNC}$ technology. While the digital beam prototype 
revealed the capabilities of this process, the design of the pavilion and developing threedimensional shell forms with this method revealed how much more research is required to refine this process even further.

Moving from the 2D, planar geometry of the first chapter to 3D shell surfaces of the second introduced an exponentially more complex geometry and corresponding analytical process. Without question the computational methodologies developed to generate the digital beam prototype are not sufficient on their own if the subject form is a complex 3D surface. Thus, the working methodology developed for the pavilion design is the initial attempt to force the working methodology of the previous chapter to evolve and adapt to more complex geometry.

The material potential of wood is immense, and this thesis has explored but a minor aspect of this. Only after stepping back and taking stock of the work completed do I realize how much more there is one can do. 


\section{Chapter III - The Forest}

The subject matter of Digital Wood: Computational Grain Realignment dealt with very specific material systems and computational processes. Investigating the anisotropic properties of wood and the potential to improve the structural capabilities of wood framing by manipulating the longitudinal axis of wood grain proved an immense undertaking. Despite the microcosmic nature of this project, it is important to acknowledge the greater context in which investigations such as this are posited. This thesis is representative of a shift in architectural discourse that has existed since computation and digital methods of design became accessible to architects.

Architectural investigation is conventionally conducted through iterative sketching or other forms of visual discovery. Rivka Oxman argues that sketching through various iterations to advance design is the antithesis of the digital method. The digital method advances only through the discovery of new knowledge, not intuition, artistic discourse, or empathetic reasoning. It is a process beyond the visual interpretation of form (Oxman, 2012). The objective nature and somewhat altruistic characterization of the digital methods (as per Oxman) are not without their criticisms. The ability to generate complex forms has lead to an abuse of computing power in a sense. Unlike any time before, there is an abundance of gratuitous, self-serving and in some cases unbuildable forms that claim to be architecture. In many cases these forms possess no material connection or rational tectonic logic. This is the blight on computation in architecture.

Digital Wood attempts to bridge the divide that exists between natural systems and the wholly unnatural principle of computation. The chapter structure established, branches, trees, the forest, metaphorically describes the nature of the content of each chapter. Branches details the process of developing individual structural components that could later be used in a larger system. Trees details the process of implementing the knowledge obtained from Branches to develop a network of structural components in the form of a research pavilion. Trees, this chapter, steps back to reflect of the greater implications of this research. This structure, Braches, Tress, Forest, is also an established convention in computation to describe data. Data trees are banks of information that can further broken down and compartmentalized to their individual branches. The true power of computation for design is not complex form generation purely for the sake of form, but the investigative ability to model and simulate natural processes. With computation architects can look to nature and

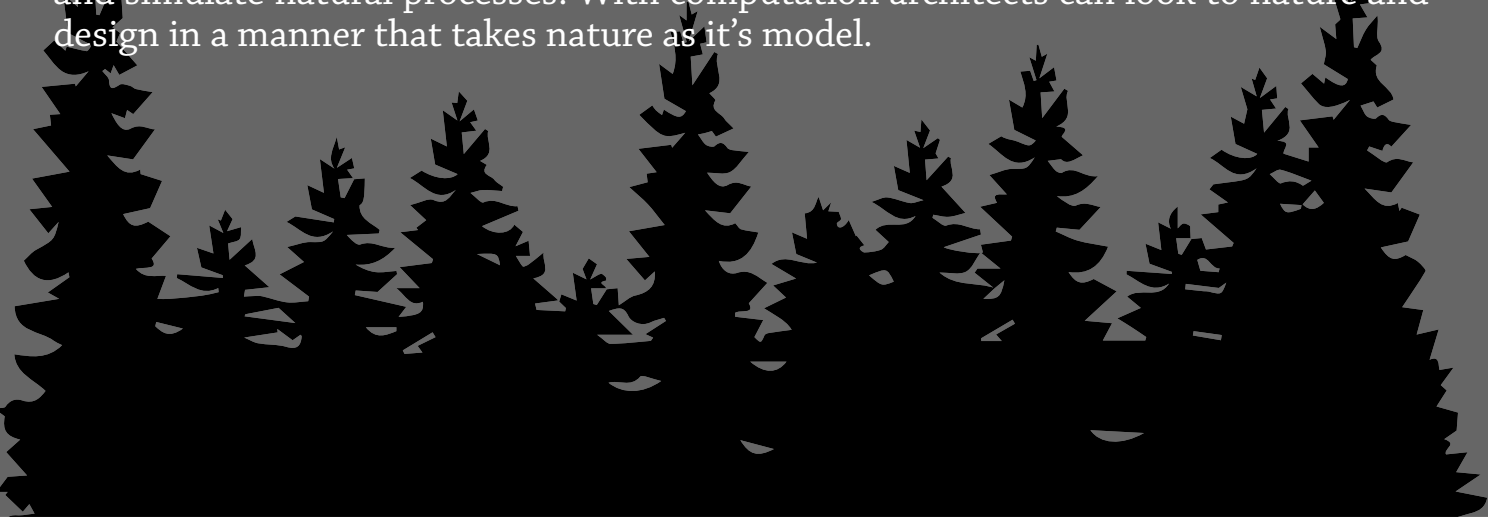




\section{Glossary of Terms}

Archi-Structure: An entwined relationship of architecture and structure where each element mutually influences and defines the other.

Clear Wood: Samples of wood with uniform anatomical characteristics (grain, growth rings, etc...) used for testing and establishing mechanical properties of a particular wood species.

Design Intelligence: Referring to a natural system that seemingly demonstrates a degree of intelligent behavior through it's interactions with the physical world. Specific to this thesis, the natural processes of grain deposition through tree growth whereby material is deposited in a manner that evenly distributes a force throughout the mass of the tree, and in doing so achieves a state of equilibrium where there are no isolated points of high internal stress.

FEA Solver: The computational engine in the Grasshopper3D environment that assimilates all system inputs and calculates that structural reactions. The solver results are affected by the mesh resolution of the design geometry and solver iterations allowed per solution.

FEA Solver Iterations: The number of complete calculations the FEA Solver is permitted to compute. Increasing the number of iterations will increase the accuracy of the solution provided.

Finite Element Analysis: Computational method of analyzing stress on complex geometries that could not otherwise be calculated with traditional analytical methods.

Grain Realignment: A computation-to-fabrication design sequence whereby internal principle stresses are isolated and utilized to generate wood-composite structural components where the longitudinal axis of the wood grain, which is strong in tension and compression, is parallel to the principle stress.

Jointer: A common machine used in fine woodworking to create a flat, true corner.

Lamination: In the context of this thesis refers to the process of gluing multiple thin layers of veneer with adhesives while clamping under pressure.

Locator Jig: A tool developed during this thesis to assist locating stock material on the bed of a CNC mill for precise milling operations. A Locator Jig establishes a common point of 
origin $(0,0,0)$ between the machine and the stock to be milled while also serving to hold the stock in-place, eliminating the need for clamping that could potential interrupt a tool path.

Mesh Geometry: A form of geometric modeling through computation whereby mathematical surfaces and solids objects are approximated by a triangular or quadrilateral surface division. A multi-faceted mesh is composed of vertices, edge curves and face surfaces and serves as a geometric approximation of the object it is describing.

Mesh Resolution: The number of individual facets that compose a mesh geometry. In addition to more closely describing a geometry visually, a higher mesh resolution allows for more accurate FEA calculations, but also requires increased computation abilities.

Polyline: Closed curve object in digital modeling common to all software applications. Very common in subtractive manufacturing to generate machine toolpaths.

Subtractive Manufacturing: Any form of milling or machining that produces a finishes object by removing material

Surface Planer: A common machine used in fine woodworking to reduce the thickness of a board. Rotary knives remove a predetermined thickness of material along the longitudinal axis of a piece of lumber.

Utility Script: A scripted component in the grasshopper environment developed to execute repetitive, numerical operations on large volumes of data. Operations of utility scripts include separating, sorting, sifting, culling, categorizing, compartmentalizing and restructuring, etc... as dictated by numerical operators and criteria within the script.

Wood-composite Assemblies: Multi-ply veneer assemblies formed and laminated with adhesives under pressure to produce a desired geometric form that satisfies a design condition.

2.5D: A common term to describe milling operations that are driven by two-dimensional linework. The objects milled are in fact three-dimensional objects in space, however this is typically the thickness of the stock material that was milled from. 


\section{Bibliography}

Botannini, Lorenzo F. (ed) (2011) Wood: Types, Properties, and Uses. New York: Nova Science Publishers.

Graubner, Wolfram (ed) (1992). Encyclopedia of Wood Joints. Newtown: Taunton Press.

Hensel, M, Menges, A, and Weinstock, M (eds) (2010). Emergent Technologies and Design. Oxon: Routledge.

Heidegger, Martin. (2008). Basic Writings - The Question Concerning Technology. New York: Harper Perennial.

Ishida, Emile H and Ryuzo Furukawa. (2013). Nature Technology: Creating a Fresh Approach to Technology and Lifestyle. Japan: Springer.

Kottas, Dimitris (ed). (2012). Architecture \& Construction: Wood. Barcelona: Linksbooks.

Mattheck, Claus. (1991). Trees: The Mechanical Design. Berlin: Springer-Verlag.

Mattheck, Claus. (1998). Design in Nature: Learning from Trees. Berlin: Springer-Verlag.

Mori, Toshiko. (ed). (2002). Immaterial / Ultramaterial: Architecture, Design, and Materials. Cambridge, Massachusetts: Harvard Design School in Association with George Braziller.

Oxman, Rivka. (2012). Chapter 2, Novel Concepts in Digital Design, Computation Design Methods. Israel: Technion - Israel Institute of Technology.

Terzidis, Kostas. (2003). Expressive Form: A Conceptual Approach to Computational Design. London: Spoon Press.

Thompson, Rob. (2011). Prototyping and Low-Volume Production. London: Thames \& Hudson.

Tsoumis, George. (ed) (1991). Science and Technology of Wood. New York: Van Nostrand Reinhold.

Wood Handbook: Wood as an Engineering Material. Ottawa, Ontario: Algrove Publishing Limited, 2002. 